\title{
Heating of blue compact dwarf galaxies: gas distribution and photoionization by stars in IZw $18^{\star}$
}

\author{
D. Péquignot
}

\author{
LUTH, Observatoire de Paris, CNRS, Université Paris Diderot; 5 place Jules Janssen, 92190 Meudon, France \\ e-mail: Daniel.Pequignot@obspm.fr
}

Received 24 July 2007 / Accepted 31 October 2007

\section{ABSTRACT}

\begin{abstract}
Aims. Photoionization models so far are unable to account for the high electron temperature $T_{\mathrm{e}}([\mathrm{O} \mathrm{III}])$ implied by the line intensity ratio [O III] $\lambda 4363 \AA /[\mathrm{O}$ III] $] \lambda 5007 \AA ̊$ in low-metallicity blue compact dwarf galaxies, casting doubt on the assumption of photoionization by hot stars as the dominant source of heating of the gas in these objects of large cosmological significance.

Methods. Combinations of runs of the 1D photoionization code NEBU are used to explore alternative models for the prototype giant $\mathrm{H}$ II region shell IZw $18 \mathrm{NW}$, with no reference to the filling factor concept and with due consideration for geometrical and stellar evolution constraints.

Results. Acceptable models for IZw $18 \mathrm{NW}$ are obtained, which represent schematically an incomplete shell comprising radiationbounded condensations embedded in a low-density matter-bounded diffuse medium. The thermal pressure contrast between gas components is about a factor 7. The diffuse phase can be in pressure balance with the hot superbubble fed by mechanical energy from the inner massive star cluster. The failure of previous models is ascribed to (1) the adoption of an inadequate small-scale gas density distribution, which proves critical when the collisional excitation of hydrogen contributes significantly to the cooling of the gas, and possibly (2) a too restrictive implementation of Wolf-Rayet stars in synthetic stellar cluster spectral energy distributions. A neutral gas component heated by soft X-rays, whose power is less than $1 \%$ of the star cluster luminosity and consistent with CHANDRA data, can explain the low-ionization fine-structure lines detected by SPITZER. [O/Fe] is slightly smaller in IZw $18 \mathrm{NW}$ than in Galactic Halo stars of similar metallicity and $[\mathrm{C} / \mathrm{O}]$ is correlatively large.

Conclusions. Extra heating by, e.g., dissipation of mechanical energy is not required to explain $T_{\mathrm{e}}$ ([O III]) in IZw 18. Important astrophysical developments depend on the 5\% uncertainty attached to [O III] collision strengths.
\end{abstract}

Key words. galaxies: individual: I Zw 18 - galaxies: starburst - ISM: H II regions - stars: early-type - stars: Wolf-Rayet atomic data

\section{Introduction}

The optical properties of Blue Compact Dwarf (BCD) galaxies are similar to those of Giant Extragalactic H II Regions (GEHIIR). Their blue continuum arises from one or several young Massive Star Clusters (MSC), which harbour extremely large numbers of massive stars.

BCDs are relatively isolated, small-sized, metal-poor galaxies (Kunth \& Östlin 2000) and may be the rare "living fossils" of a formerly common population. BCDs can provide invaluable information about the primordial abundance of helium (e.g., Davidson \& Kinman 1985), the chemical composition of the InterStellar Medium (ISM, e.g., Izotov et al. 2006a), the formation and evolution of massive stars, and the early evolution of galaxies at large redshift. Among them, IZw 18 stands out as one of the most oxygen-poor BCDs known (e.g., Izotov et al. 1999) and a young galaxy candidate in the Local Universe (e.g., Izotov \& Thuan 2004).

The line emission of H II regions is believed to be governed by radiation from massive stars, but spectroscopic diagnostics most often indicate spatial fluctuations of the electron temperature $T_{\mathrm{e}}$ (see the dimensionless parameter $t^{2}$, Peimbert 1967) that appear larger than those computed in usual photoionization models, suggesting an extra heating of the emitting gas (e.g.,

* Appendices are only available in electronic form at http: //www . aanda.org
Peimbert 1995; Luridiana et al. 1999). Until the cause(s) of this failure of photoionization models can be identified, a basic tool of astrophysics remains uncertain.

Tsamis \& Péquignot (2005) showed that, in the GEHIIR 30 Dor of the LMC, the various $T_{\mathrm{e}}$ diagnostics could be made compatible with one another if the ionized gas were chemically inhomogeneous over small spatial scales. A pure photoionization model could then account for the spectrum of a bright filament of this nebula. Although this new model needs confirmation, it is in agreement with the scenario by Tenorio-Tagle (1996) of a recycling of supernova ejecta through a rain of metal-rich droplets cooling and condensing in the Galaxy halo, then falling back on to the Galactic disc and incorporating into the ISM without significant mixing until a new $\mathrm{H}$ II region eventually forms. If this class of photoionization model is finally accepted, extra heating will not be required for objects like 30 Dor, with near Galactic metallicity.

Another problem is encountered in low-metallicity ("lowZ") BCDs (Appendix A). In BCDs, available spectroscopic data do not provide signatures for $t^{2}$, but a major concern of photoionization models is explaining the high temperature $T_{\mathrm{e}}([\mathrm{O} \mathrm{III}])$ infered from the observed intensity ratio $r([\mathrm{O} \mathrm{III}])=$ [O III $] \lambda 4363 /([\mathrm{O}$ III $] \lambda$ 5007+4959). Thus, Stasińska \& Schaerer (1999, SS99) conclude that photoionization by stars fails to explain $r([\mathrm{O} \mathrm{III}])$ in the GEHIIR IZw $18 \mathrm{NW}$ and that photoionization must be supplemented by other heating mechanisms. 
A requirement for extra heating is indirectly stated by Luridiana et al. (1999) for NGC 2363.

A possible heating mechanism is conversion of mechanical energy provided by stellar winds and supernovae, although a conclusion of Luridiana et al. (2001) is not optimistic. A limitation of this mechanism is that most of this mechanical energy is likely to dissipate in hot, steadily expanding superbubbles (Martin 1996; Tenorio-Tagle et al. 2006). It is doubtful that heat conduction from this coronal gas could induce enough localized enhancement of $T_{\mathrm{e}}$ in the photoionized gas (e.g., Maciejewski et al. 1996), even though Slavin et al. (1993) suggest that turbulent mixing may favour an energy transfer. Martin (1997) suggests that shocks could help explain the trend of ionization throughout the diffuse interstellar gas of BCDs, but concedes that "shocks are only being invoked as a secondary signal in gas with very low surface brightness". Finally, photoelectric heating from dust is inefficient in metal-poor hot gas conditions (Bakes \& Tielens 1994).

Nevertheless, the conclusion of SS99 is now accepted in many studies of GEHIIRs. It entails such far-reaching consequences concerning the physics of galaxies at large redshifts as to deserve close scrutiny. If, for exemple, the difference between observed and computed $T_{\mathrm{e}}([\mathrm{O} \mathrm{III}])$ in the model by SS99 were to be accounted for by artificially raising the heat input proportionally to the photoionization heating, then the total heat input in the emitting gas should be doubled. This problem therefore deals with the global energetics of the early universe.

After reviewing previous models for IZw $18 \mathrm{NW}$ (Sect. 2), observations and new photoionization models are described in Sects. 3 and 4. Results presented in Sect. 5 are discussed in Sect. 6. Concluding remarks appear in Sect. 7. Models for other GEHIIRs are reviewed in Appendix A. Concepts undelying the new photoionization models are stated in Appendices B and C.

\section{Photoionization models for I Zw 18 NW}

Early models are reviewed by SS99. Dufour et al. (1988) envisioned a collection of small $\mathrm{H}$ II regions of different excitations. Campbell (1990) proposed to enhance $r([\mathrm{O}$ III] $)$ by collisional quenching of [O III] $\lambda 5007$ in an ultra-compact structure (electron density $N_{\mathrm{e}}=10^{5} \mathrm{~cm}^{-3}$ ). Stevenson et al. (1993) modelled a uniform sphere of radius $\sim 0.4^{\prime \prime}$. Fairly satisfactory computed emission-line spectra were obtained, but the model $\mathrm{H}$ II regions were inacceptably compact according to subsequent imaging. Firstly, I Zw $18 \mathrm{NW}$ is essentially an incomplete H II region shell of some $5^{\prime \prime}$ in diameter surrounding a young MSC, which is not spatially coincident with the ionized gas. Secondly, both the highly $T_{\mathrm{e}}$-sensitive line [O III] $\lambda 4363$ and the high-ionization line He II $\lambda 4686$ are detected throughout the shell and beyond.

\subsection{Recent modelling attempts}

According to SS99, the "model" is a uniform, matter-bounded spherical shell whose only free parameter is a filling factor $\epsilon$. The hydrogen density is $N_{\mathrm{H}}=10^{2} \mathrm{~cm}^{-3}$, obtained by the electron density $N_{\mathrm{e}}([\mathrm{S} \mathrm{II}])$ derived from the observed doublet intensity ratio $r([\mathrm{~S}$ II $])=[\mathrm{S} \mathrm{II}] \lambda 6716 /[\mathrm{S}$ II $] \lambda 6731$. The central ionizing source is a synthetic stellar cluster which fits the observed continuum flux at $3327 \AA$ and maximizes the nebular He II emission. The inner angular radius is $1.5^{\prime \prime}$. The outer radius $r_{\text {out }}$ is defined by the condition that the computed [O III $] \lambda 5007 /[\mathrm{O}$ II $] \lambda 3727$ ratio fits the observed one. For increasing $\epsilon$, the material is on average closer to the source and more ionized, which must be compensated for by increasing the optical depth to keep $[\mathrm{O}$ III $] /[\mathrm{O}$ II $]$ constant, so that the computed $\mathrm{H} \beta$ flux increases and $\mathrm{He} \mathrm{I} / \mathrm{H} \beta$ decreases. For $\epsilon>0.1$, the shell becomes radiation bounded, [O III]/[O II] grows larger than it should and $r_{\text {out }}$ becomes less than the observed value $\left(\sim 2.5^{\prime \prime}\right)$. Because of these trends, SS99 discard large- $\epsilon$ models and select a model with $\epsilon \sim 0.01$, on the basis that the computed $r_{\text {out }}, \mathrm{H} \beta$ and He II are roughly acceptable". This "best model" presents two major drawbacks: (1) as stated by SS99, the computed $r([\mathrm{O}$ III]) is too small by a highly significant factor of 1.3 , and (2) [S II] and [O I] are grossly underestimated.

\subsection{The [OIII] line problem}

Concerning $r([\mathrm{O} \mathrm{III}])$, SS99 note without justification that, for different values of $N_{\mathrm{H}}$, "no acceptable solution is found". This is central in concluding that extra heating is required.

In response, Viegas (2002, hereafter V02) states that adopting a density less than $10^{2} \mathrm{~cm}^{-3}$ (and $\epsilon=1$ ) can help improve the computed $r([\mathrm{O} \mathrm{III}])$. However, in the example shown by $\mathrm{V} 02$ $\left(N_{\mathrm{e}}=30 \mathrm{~cm}^{-3}\right), r([\mathrm{O} \mathrm{III}])$ is still $10 \% \mathrm{low}^{2}$ and $r([\mathrm{~S} \mathrm{II}])$ is not accurate. Moreover, not only [S II] and [O I], but now [O II] as well is strongly underpredicted, in accordance with the analysis of SS99. V02 then proposes that radiation-bounded filaments with density $10^{4} \mathrm{~cm}^{-3}$ are embedded in the low-density gas at different distances from the source. If the emission of [S II] and [OI] (together with [O II]) can be increased in this way, this denser component has difficulties. Firstly, since the computed [O III]/[O II] ratio is not very much less than the observed one in these filaments, a sizeable fraction of [O III] must come from them (together with [O II]) and since, due to enhanced H I cooling (Appendix B.1), $r([\mathrm{O}$ III $]$ ) is now half the observed value, any composite model accounting simultaneously for [O III $] \lambda 5007$ and [O II] $\lambda 3727$ will underpredict $r([\mathrm{O}$ III $])$ in the same manner as the uniform model of SS99. Secondly, since at least half the [S II] emission should come from the filaments in which $r([\mathrm{~S} \mathrm{II}])$ is again only half the observed value (large $N_{\mathrm{e}}$ ), the composite $r([\mathrm{~S}$ II $])$ will be inacceptably inaccurate. Thirdly, no explicit solution is found and it is unclear how a composite model of the kind envisaged by V02 will simultaneouly match all lines. From the evidence, the claim of V02 that "pure photoionization can explain IZw 18 observations" is not supported. The inconclusiveness of the alternative she proposes effectively reinforces the standpoint of SS99.

\subsection{The [OI] and [SII] line problem}

[O I] $\lambda 6300+63$ is underpredicted by 2 dex in the model of SS99. Two configurations are envisaged by SS99.

\subsubsection{Extremely dense filaments?}

In a first configuration, radiation-bounded filaments of density $10^{6} \mathrm{~cm}^{-3}$ are embedded in the $\mathrm{H}$ II region: the density is so high as to severely quench most lines other than [OI] and H I: only $\sim 10 \%$ of $[\mathrm{S} \mathrm{II}] \lambda 6716+31$ arises from these filaments,

\footnotetext{
1 In this context, the $\mathrm{H} \beta$ and $\mathrm{He}$ II fluxes are poor selection criteria for $\epsilon$, since the former is proportional to the (unknown) covering factor of the shell and the latter depends much on questionable synthetic stellar cluster spectra (Appendix C).

2 The tolerance of $9 \%$ allowed by SS99 (adopted by V02) is probably too large (Sect. 3.3). This error bar is justified in the logic of SS99, who aim to demonstrate an absence of solution.
} 
ensuring that $r([\mathrm{~S} \mathrm{II}])$ is not much influenced. This attempt to solve in anticipation the problem met by V02 (Sect. 2.2) raises three difficulties, however: (1) condensations that contrast in density by a factor of $10^{4}$ with their surroundings and present a large enough covering factor ( $\sim 10 \%$ according to SS99) as to intercept a significant fraction of the primary radiation would probably represent most of the mass; (2) since the main body of the model $\mathrm{H}$ II region produces only one quarter of the observed [S II] $\lambda 6716+31$ flux, it is not clear where this doublet would be emitted $^{3}$; and (3) this highly artificial, strictly dual density distribution is not the schematic, first-approximation representation of some more complex reality, rather it is an essential feature of the model since any material at intermediate densities would usefully emit [S II] $\lambda 6716+31$, but lead to a totally wrong $r$ ([S II] $)$, as in the description by V02.

\subsubsection{Extremely distant filaments?}

The second configuration proposed by SS99 involves radiation bounded "[O I ] filaments" of density $10^{2} \mathrm{~cm}^{-3}$, located at $\sim 20^{\prime \prime}$ from the source. If the spectroscopic objections of Sects. 2.2 and 2.3.1 are now removed since density is moderate and ionization is low in the filaments, new difficulties arise, notably with geometry: (1) the filaments observed at $>10^{\prime \prime}$ or more from the NW MSC of IZw 18 have such a low surface brightness as to contribute negligibly to the brightness of the main shell (if they were projected upon it); (2) the spectrum of this weak emission up to 15" - "Halo" of Vílchez \& Iglesias-Páramo (1998), "H $\alpha$ Arc" and "Loop" of Izotov et al. (2001a) - shows a flux ratio [O III] $\lambda 5007 /[\mathrm{O}$ II $] \lambda 3727$ of order unity, whereas this ratio is $1 / 300$ in the putative [O I] filaments, suggesting that the bulk of the emission observed at these distances arises from a gas whose density is much less than $10^{2} \mathrm{~cm}^{-3}$; (3) accepting all the same the existence of distant [O I] emitting regions, a very peculiar geometry would be required to project these regions precisely and uniquely upon the material of the irregular bright NW H II shell to be modelled; and (4) in projection, this shell appears as a $1.5-2.5$ " "ring", which intercepts $1 / 200$ of a 20 "-radius sphere (including both the front and rear sides), incommensurable with the covering factor $\sim 1 / 10$ assumed by SS99.

\subsection{Previous models: conclusion}

Attempts to model IZw 18 fail to explain not only $r$ ([O III] $]$ but the [O I] and [S II] lines as well. It is difficult to follow SS99 when they claim that they are "not too far from a completely satisfactory photoionization model" of I Zw 18 . The explanatory value of their description is so loose as to jeopardize any inference drawn from it, including the requirement for extra heating in IZw 18.

In Appendix A, a review of models obtained for other GEHIIRs reveals general trends and problems, which can be valuably analysed using the example of IZw 18.

\section{Observations of IZw 18}

\subsection{Basic properties}

Two bright regions 5" apart, IZw $18 \mathrm{NW}$ and SE, correspond to two young MSCs associated with two distinct GEHIIRs, surrounded by a common irregular, filamentary halo of diffuse ionized gas (e.g., Izotov et al. 2001a), immersed in a radio H I $21 \mathrm{~cm}$

\footnotetext{
${ }^{3}$ SS99 prefer casting doubt on the ionization balance of $\mathrm{S}^{+}$.
}

envelope rotating around the centre of mass located in between the GEHIIRs (e.g., van Zee et al. 1998). Although the H I column density peaks in the central region, large $\mathrm{H}$ I structures have no stellar counterparts. A fainter cluster, "Component C", deprived of massive stars (no prominent $\mathrm{H}$ II region), appears at 22 " to the NW of the main body. The two young MSCs, 1-5 Myr old, are the recent manifestations of a larger starburst, which started some $15 \mathrm{Myr}$ ago in Component $\mathrm{C}$ and $20 \mathrm{Myr}$ ago in the central region (Izotov \& Thuan 2004, IT04). A 20-25 Myr age is consistent with the dynamics of the superbubble studied by Martin (1996). In a radio study, Hirashita \& Hunt (2006) suggest 12-15 Myr. IZw 18 is classified as a "passive BCD" (e.g., Hirashita \& Hunt 2006), that is, the MSCs themselves are relatively diffuse, the stellar formation rate (SFR) is relatively low (Sect. 6.2) and the starburst is not instantaneous.

That a background population 300-500 Myr old may be the first generation of stars in this galaxy (Papaderos et al. 2002; IT04) is contested by Aloisi et al. (2007). The extended optical halo of I Zw 18 is mostly due to ionized gas emission. Unlike for usual BCDs, the bulk of the stars in IZw 18 is highly concentrated, suggesting perhaps a young structure (Papaderos et al. 2002). The distance to I Zw 18 , first quoted as $10 \mathrm{Mpc}$, has been revised to $\sim 13 \mathrm{Mpc}$ (Östlin 2000) after correcting the Hubble flow for the attraction of the Virgo cluster. From AGB star magnitudes, IT04 obtain $14 \pm 1.5 \mathrm{Mpc}$. At the distance $D=$ $4.0 \times 10^{25} \mathrm{~cm}(12.97 \mathrm{Mpc})$ adopted here, the diameter of the bright region IZw $18 \mathrm{NW}\left(5^{\prime \prime}\right)$ is over $300 \mathrm{pc}$. From new deep HST photometry revealing a red giant branch and Cepheid variables, Aloisi et al. (2007) obtain $18 \pm 2$ Mpc. Except for scaling, present results are just marginally changed if this larger distance is confirmed (Sect. 6.8).

\subsection{Absolute $H I$ line fluxes and reddening}

According to Cannon et al. (2002, CSGD02), the absolute $\mathrm{H} \beta$ fluxes in the 5 polygons paving the NW region and the 7 polygons paving the SE region are 4.9 and 1.7 respectively in units of $10^{-14} \mathrm{erg} \mathrm{cm}^{-2} \mathrm{~s}^{-1}$. Polygons NW D6 and SE D8 do not exactly belong to the main body of the H II regions and are dismissed. Tenuous emission around the polygons is also neglected.

The excess over the Case B recombination value of the observed average $\mathrm{H} \alpha / \mathrm{H} \beta$ ratios, 2.94 and 2.97 in the NW and SE respectively, is attributed to dust reddening by CSGD02, who rightly doubt the large $\mathrm{HI}$ collisional excitation obtained by SS99 (Appendix D.1). It remains that the Balmer decrement is influenced by collisions and that the reddening correction to the observed spectrum of I Zw 18 has been overestimated. Collisional excitation results from a subtle anticorrelation between $T_{\mathrm{e}}$ and $\mathrm{N}\left(\mathrm{H}^{0}\right) / \mathrm{N}\left(\mathrm{H}^{+}\right)$within the nebula and can only be determined from a photoionization model (contrary to a statement by CSGD02, the maximum effect does not correspond to the hottest gas). The usually adopted recombination ratio is $\mathrm{H} \alpha / \mathrm{H} \beta=2.75 \pm 0.01$ (Izotov et al. 1999). It is anticipated that, according to present models (Sect. 5), a better $\mathrm{H} \alpha / \mathrm{H} \beta$ is $2.83 \pm$ 0.02 . For use in the present study, published dereddened intensities (also corrected for stellar absorption lines) have been rereddened by $\Delta E(B-V)=-0.04$ (in view of final results, a more nearly accurate correction could be $\Delta E(B-V)=-0.03)$. Then the typical $E(B-V)$ for IZw $18 \mathrm{NW}$ shifts from 0.08 to 0.04 , out of which the foreground Galactic contribution is about 0.02 (Schlegel et al. 1998). The reddening corrected $\mathrm{H} \beta$ fluxes for the main NW and SE H II regions are $I(\mathrm{H} \beta)=5.6$ and 2.0 respectively in units of $10^{-14} \mathrm{erg} \mathrm{cm}^{-2} \mathrm{~s}^{-1}$. 
In these units, the $\mathrm{H} \alpha$ flux is 33.0 over the central $13.7 \times$ 10.5" HST field (Hunter \& Thronson 1995; CSGD02) and 42.0 over a $60 \times 60^{\prime \prime}$ field (Dufour \& Hester 1990). Adopting overall averages $\mathrm{H} \alpha / \mathrm{H} \beta=2.8$ and $E(B-V)=0.06$, the total dereddened $\mathrm{H} \beta$ flux for I Zw 18 is 18.3 . Assuming that all of the ionizing photon sources belong to the bright NW and SE MSCs and that IZw 18 is globally radiation bounded, the fraction of photons absorbed in the two main H II regions is 0.41 . Let $Q$ and $Q_{\mathrm{abs}}$ be respectively the number of photons $\left(\mathrm{s}^{-1}\right)$ emitted by the MSC and absorbed by the main shell of I Zw $18 \mathrm{NW}$ alone. The fraction $Q_{\mathrm{abs}} / Q$ may be smaller than 0.41 for two reasons. Firstly, as expansion proceeds, the shells around the starbursts become more "porous" due to instabilities and the more evolved NW shell may be more affected. Assuming that no photons escape from the SE shell leads to a minimum $Q_{\mathrm{abs}} / Q=0.34$. A more realistic value is probably $Q_{\mathrm{abs}} / Q=0.39 \pm 0.02$, since a complete absorption in the SE would result in a strong asymmetry of the diffuse halo, which is not observed. Secondly, photons may escape from I Zw 18 . This effect is probably weak, given the amount and extension of $\mathrm{H}$ I in I Zw 18 . The adopted nominal absorbed fraction for the NW shell will be $Q_{\mathrm{abs}} / Q=0.37 \pm 0.03$, with 0.30 a conservative lower limit, obtained for a $25-30 \%$ escape from I Zw 18.

\subsection{Spectroscopic observation summary}

The optical spectrum of IZw 18 has been observed for decades (Sargent \& Searle 1970; Skillman \& Kennicutt 1993, SK93; Legrand et al. 1997; Izotov et al. 1997a,b; Izotov \& Thuan 1998; Vílchez \& Iglesias-Páramo 1998, VI98; Izotov et al. 1999, ICF99; Izotov et al. 2001a; Thuan \& Izotov 2005, TI05; Izotov et al. 2006a) with many instruments (HALE, KPNO, MMT, KECK, CFHT, etc.), the UV spectrum with IUE (Dufour et al. 1988) and HST (Garnett et al. 1997; Izotov \& Thuan 1999, IT99), the IR spectrum with SPITZER (Wu et al. 2006, 2007), and the radio continuum with VLA (Hunt et al. 2005; Cannon et al. 2005).

The IUE aperture encompasses all of the bright regions. In addition to $\mathrm{C}$ III] $\lambda 1909$, there are indications for the presence of C IV $\lambda 1549$ and Si III] $\lambda 1883+92$. The HST spectrum allows a direct comparison of $\mathrm{C}$ III] with optical lines, but corresponds to such a limited area $\left(0.86^{\prime \prime}\right)$ as to raise the question of the representativeness of the observation for IZw $18 \mathrm{NW}$ as a whole. Nonetheless, $\mathrm{C} \mathrm{III]/H} \beta$ is identical within $10 \%$ in available measurements, once the re-evaluation of the $\mathrm{H} \alpha$ flux within the IUE aperture is taken into account (Dufour \& Hester 1990).

The high-resolution mid-IR spectra of IZw 18 (Wu et al. $2007, \mathrm{Wu} 07$ ) are secured with a $4.7 \times 11.3^{\prime \prime}$ slit. Over the $13.7 \times$ $10.5^{\prime \prime}$ HST field, the de-redenned $\mathrm{H} \beta$ flux is 13.4 , while the flux from strictly the two central H II regions, which fill only part of the SPITZER slit, is 7.6. The adopted $\mathrm{H} \beta$ flux corresponding to the mid-IR spectra is taken as $10 \pm 1$, the value also used by Dufour \& Hester (1990) for the (partial) IUE aperture. Measuring line fluxes on the published tracings shows excellent agreement with tabulated values, except for [S III] $18.7 \mu$, whose flux is tentatively shifted from 2.3 to $2.8 \times 10^{-15} \mathrm{erg} \mathrm{cm}^{-2} \mathrm{~s}^{-1}$. The UV and mid-IR spectra are not fully specific to I Zw $18 \mathrm{NW}$.

An average de-reddened emission line spectrum for I Zw $18 \mathrm{NW}$, close to the one secured by ICF99 in the optical range, is presented in Col. 2 ("Obs.") of Table 3 (line identifications in Col. 1; Cols. 3-6 are presented in Sect. 5). This spectrum differs little from those by Izotov \& Thuan (1998) and SK93. A rather deep, high-resolution red spectrum is presented by SK93. A few weak lines are taken from a deep blue MMT spectrum by TI05, who however quote an [O II] 3727 flux larger than in earlier studies. Absolute fluxes for $\mathrm{H} \beta$ and the radio continuum are given on top of Table 3 . The $21 \mathrm{~cm}$ and $3.6 \mathrm{~cm}$ fluxes, obtained from Cannon et al. (2005) as a sum of 3 contours for the NW shell, partly originate in non-thermal processes, not considered here. Line intensities are relative to $\mathrm{H} \beta=$ 1000 . The intensity ratio [N II] $\lambda 6584 / \lambda 6548$ quoted by SK93 is smaller than the theoretical value: this is presumably due to the presence of a broad $\mathrm{H} \alpha$ component (VI98). Correcting for the pseudo-continuum, the theoretical ratio is recovered and a new, smaller value is obtained for the sum of the [N II] doublet. [O II] 7320+30 (SK93) is uncertain and difficult to link to $\mathrm{H} \alpha$. Taking into account weak (undetected) lines, such as [Ne IV] $\lambda$ 4724, [Fe III] $\lambda \lambda 4702,4734,4755$, a continuum slightly lower than the one adopted by TI05 leads to a moderate increase of the [Ar IV] line fluxes. Lines [Fe IV] 4906, [Fe II] 5158 and [Fe VI] 5176 are seen in the tracing by IT05, with tentative intensities 3, 2 and $2(\mathrm{H} \beta=1000)$ respectively. Only [Fe IV] is considered in Table 3 (It is noted that the predicted intensities for these [Fe II] and [Fe VI] lines will be $\sim 1)$.

The most critical (de-reddened) line ratio is $r([\mathrm{O} \mathrm{III}])=$ 0.0246 , the value also adopted by SS99. This is $3.1 \%$ larger than the often quoted value by SK93 ( $2^{\prime \prime}$ slit), $0.6 \%$ smaller than the value by ICF99 (1.5" slit) and $2.3 \%$ smaller than in the blue spectrum by TI05 (2" slit).

\section{New photoionization models for IZw 18 NW}

Models are computed using the standard photoionization code NEBU (Péquignot et al. 2001) in spherical symmetry with a central point-like source, suited to the apparent geometry of I Zw $18 \mathrm{NW}$ since the bulk of the stars of the NW MSC belongs to a cavity surrounded by the GEHIIR shell. Radiation-bounded filaments embedded in a diffuse medium are modelled. The reader is referred to Appendices $\mathrm{B}$ and $\mathrm{C}$ for a perspective to the present approach. Atomic data are considered in Appendix D.

\subsection{Stellar ionizing radiation}

The central source Spectral Energy Distribution (SED) is treated analytically, with no precise reference to existing synthetic stellar cluster SEDs (Appendix C). No effort is made to describe the optical+UV continuum. The continuum flux at $\lambda 3327 \AA$ (de Mello et al. 1998) is not used to constrain the power of the MSC. Here, this constraint can be replaced to great advantage by the fraction of ionizing photons absorbed in the shell (Sect. 3.2).

The continuous distribution of stellar masses most often results in an approximately exponential decrease of flux with photon energy from 1 to 4 ryd in the SED of current synthetic MSCs (e.g., Luridiana et al. 2003). The sum of two black bodies at different temperatures can mimic this shape, yet provide flexibility to study the influence of the SED. The source of ionizing radiation is described as the sum of a hot black body, BB1 (temperature $T 1 \geq 60 \mathrm{kK}$; luminosity $L 1$ ), and a cooler one, BB2 $(T 2=40-50 \mathrm{kK} ; L 2)$. A constant scaling factor $\delta_{4}(\leq 1)$, reminiscent of the discontinuity appearing in the SED of model stars (e.g., Leitherer et al. 1999) and constrained by the observed intensity of He II $\lambda 4686$, is applied to the BB1 flux at $\geq 4$ ryd. The ionizing continuum depends on five free parameters. The adopted $T 2$ range is reminiscent of massive main sequence stars and lower $T 2$ s need not be considered. A sufficiently large range of $T 1$ values should be considered, as the high-energy tail of the intrinsic SED is influenced by WR stars, whose properties are either uncertain or unknowable (Appendix C). 


\subsection{Ionized shell}

The IZw $18 \mathrm{NW}$ shell extends from $R_{\mathrm{i}}=2.85 \times 10^{20} \mathrm{~cm}$ to $R_{\mathrm{f}}=$ $4.75 \times 10^{20} \mathrm{~cm}\left(1.5^{\prime \prime}\right.$ and $2.5^{\prime \prime}$ at $\left.D=4.0 \times 10^{25} \mathrm{~cm}\right)$.

In final complete models a smooth small-scale density distribution is assumed (gas filling factor $\epsilon$ unity). The gas density is defined by means of the following general law for a variable gas pressure $P$, given as a function of the radial optical depth, $\tau$, at $13.6 \mathrm{eV}$ :

$P(\tau)=\frac{P_{\text {out }}+P_{\text {in }}}{2}+\frac{P_{\text {out }}-P_{\text {in }}}{\pi} \tan ^{-1}\left[\kappa \log \left(\frac{\tau}{\tau_{\mathrm{c}}}\right)\right]$.

This law is a convenient tool to explore the effects of the density distribution on the model predictions. $P$ is related to the pair of $\left(T_{\mathrm{e}}, N_{\mathrm{H}}\right)$ via the ideal gas law, with $T_{\mathrm{e}}$ derived solving the statistical equilibrium equations at each step. At the first step of the computation $(\tau=0)$, the initial pressure is $P_{\text {in }}$, while at the last step $\left(\tau=\tau_{\mathrm{m}} \sim \infty\right)$ the final pressure is $P_{\text {out }}$. A smooth, rapid transition is obtained by adopting $\kappa=30$ in all computations. Equation (1) introduces three free parameters: $P_{\text {in }}, P_{\text {out }}$, and the optical depth $\tau_{\mathrm{c}}$ at which the transition from inner to outer pressure occurs. The picture of a filament core embedded in a dilute medium dictates that $P_{\text {in }}<P_{\text {out }}$. Each filament produces a radial shadow, which emits much less than the material in front of the filament and the filament itself, since it is only subject to the weak, very soft, diffuse field from the rest of the nebula. The shadows are neglected.

In order to represent radiation-bounded filaments embedded in a low density medium (Appendix B.1), at least two sectors are needed: a "Sector 1" with $\tau_{\mathrm{m}} \gg \tau_{\mathrm{c}}$ (radial directions crossing a filament) and a "Sector 2 " with $\tau_{\mathrm{m}}<\tau_{\mathrm{c}}$. To first order, only two sectors are considered. Observation shows that the He II emission, although definitely extended, is relatively weaker in the filaments surrounding the main shell (VI98; Izotov et al. 2001a). This deficit of He II, unrelated to an outward decrease of the ionization parameter since He II is a pure "photon counting" line above 4 ryd, suggests instead that in no radial direction is the main shell totally deprived of absorbing gas. With the concern of reaching a more significant description, the same small $\tau_{\mathrm{m}}(3)=$ 0.05 will be attached to the remaining "Sector 3 " required to make up the covering factor of the source to unity in all complete models. The emission of Sector 3, a moderate contribution to the He II intensity, does not impact on conclusions concerning the main shell and the source.

For simplicity, in any given run, the values of the three defining parameters of Eq. (1) are assumed to be shared by all three sectors. Note that $\tau_{\mathrm{c}}$ and $P_{\text {out }}$ act only in Sector 1 . The topology (Appendix B.2) of the model shell is determined by giving in addition the covering factors $f_{1}^{\text {cov }}$ of Sector 1 (radiation-bounded) and $f_{2}^{\text {cov }}$ of Sector 2 (matter-bounded), with the condition:

$f_{3}^{\mathrm{cov}}=1-\left(f_{1}^{\mathrm{cov}}+f_{2}^{\mathrm{cov}}\right)>0$

and finally the optical depth $\tau_{\mathrm{m}}(2)\left(<\tau_{\mathrm{c}}\right)$ of Sector 2 . The full model shell structure depends on six free parameters.

Adopting the same $R_{\mathrm{i}}$ and the same parameters for $P(\tau)$ in the three sectors and assuming that the outer radius of Sector 1 is $R_{\mathrm{f}}$ make the computed outer radii of other sectors smaller than $R_{\mathrm{f}}$. If, however, one would like Sector 2 to extend up to $R_{\mathrm{f}}$ and perhaps beyond, models should be re-run for this sector using a $P(\tau)$ with $P_{\text {out }}(2)<P_{\text {in }}$ and $\tau_{\mathrm{c}}<1$. No significant consequences for the computed spectrum result from this change, as the increase of radius and the decrease of density in the outermost layers of Sector 2 (say, $\tau \sim 1$ ) have opposite effects on the "local" ionization. Also, "improving" the artificial geometry of Sector 3
Table 1. Constraints on model parameters ${ }^{a}$.

\begin{tabular}{|c|c|}
\hline Parameter & Constraints \\
\hline$E(B-V)$ & $\mathrm{H} \alpha / \mathrm{H} \beta ;($ No freedom: $E(B-V)=0.04)$ \\
\hline$R_{\mathrm{i}}$ & No freedom $R_{\mathrm{i}}=2.85 \times 10^{20} \mathrm{~cm}$ \\
\hline$f^{\text {cov }}$ & Absolute $I(\mathrm{H} \beta)=5.6 \times 10^{-14} \mathrm{erg} \mathrm{cm}^{-2} \mathrm{~s}^{-1}$ \\
\hline$L 1, L 2$ & Cluster SED; $f^{\text {cov }} \leq 1.0 ; Q_{\mathrm{abs}} / Q \sim 0.37$ \\
\hline$T 1, T 2$ & $\delta_{4} \leq 1.0 ;\left(\log \left(Q_{\mathrm{He}} / Q\right) \sim-0.5\right)$ \\
\hline$\delta_{4}$ & He II $\lambda 4686$ \\
\hline $\mathrm{He}$ & $\mathrm{He} / \mathrm{H}=0.08 ; \mathrm{He} \mathrm{I} \lambda 5876 ?$ \\
\hline $\mathrm{C}$ & C III] 1909 \\
\hline $\mathrm{N}$ & {$[\mathrm{N}$ II $] \lambda 6584$} \\
\hline $\mathrm{O}$ & {$[\mathrm{O} \mathrm{III}] \lambda 5007$} \\
\hline $\mathrm{Ne}$ & {$[\mathrm{Ne}$ III $] \lambda 3869$} \\
\hline $\mathrm{Mg}$ & $\mathrm{Mg} / \mathrm{Ar}=10 . ; \mathrm{Mg} \mathrm{I}] \lambda 4571 ?$ \\
\hline $\mathrm{Al}$ & $\mathrm{Al} / \mathrm{Ar}=1 . ; \mathrm{Al} \mathrm{III} \lambda 1855 ?$ \\
\hline $\mathrm{Si}$ & $\mathrm{Si} / \mathrm{Ar}=10 . ; \mathrm{Si}$ III $] \lambda 1883 ?$ \\
\hline $\mathrm{S}$ & $\mathrm{S} / \mathrm{Ar}=4.37 ;\langle[\mathrm{S} \mathrm{IIII}]\rangle ?$ \\
\hline $\mathrm{Ar}$ & {$[$ Ar III] $] 7135$} \\
\hline $\mathrm{Fe}$ & {$[\mathrm{Fe}$ III $] \lambda 4658$} \\
\hline \multicolumn{2}{|c|}{ One-component constant density run $(N 0, N 1)$ : } \\
\hline$N_{\mathrm{H}}$ & $r([\mathrm{~S} \mathrm{II}])=[\mathrm{S} \mathrm{II}] \lambda 6716 /[\mathrm{S} \mathrm{II}] \lambda 6731( \pm)$ \\
\hline$\tau_{\mathrm{m}}$ & {$[\mathrm{O}$ II] $] \lambda 3727$} \\
\hline$\epsilon$ & $R_{\mathrm{f}}=4.75 \times 10^{20} \mathrm{~cm}$ \\
\hline \multicolumn{2}{|c|}{ One-component model with Eq. (1) (M1): } \\
\hline$\epsilon=1.0$ & No freedom \\
\hline$P_{\text {out }}$ & $r([\mathrm{~S} \mathrm{II}])$ \\
\hline$\tau_{\mathrm{c}}$ & {$[\mathrm{O}$ II $] \lambda 3727$} \\
\hline$P_{\text {in }}$ & $R_{\mathrm{f}}=4.75 \times 10^{20} \mathrm{~cm}$ \\
\hline \multicolumn{2}{|c|}{ Two-component model $(M 2, M 3, M 4)$ added freedoms: } \\
\hline$f_{1}^{\operatorname{cov}}$ & $f_{2}^{\mathrm{cov}}>0 ; f^{\mathrm{cov}}=f_{1}^{\mathrm{cov}}+f_{2}^{\mathrm{cov}}<1$ \\
\hline$f_{2}^{\operatorname{cov}}$ & $\tau_{\mathrm{m}}(2)<\tau_{\mathrm{c}}$; global 3D geometry \\
\hline$\tau_{\mathrm{m}}(2)$ & Fine tuning $I(\mathrm{H} \beta)$ for given $f_{i}^{\text {cov }}$ \\
\hline
\end{tabular}

(a thin shell at radius $R_{\mathrm{i}}$ ) by assuming a lower $P_{\text {in }}$ (3) or else a filling $\epsilon \ll 1$ would not change the intensity of He II at all, while the emission of other lines from this sector is negligible.

Although three sectors are considered, Sector 3 is of no practical consequence for the main shell and no parameter is attached to it. A model based on the above description will be termed a "two-sector model" (Sect. 5.3).

\subsection{Model parameters and constraints}

Correspondances between model parameters and constraints are outlined in Table 1. The parameters are interrelated and iterations are needed to converge to a solution. The weak dependance of $E(B-V)$ on the model Balmer decrement (Sect. 3.2) is neglected. The SED is not fully determined by the major constraint $Q_{\text {abs }} / Q$. Other constraints are in the form of inequalities, some are semi-quantitative or deal with "plausibility" arguments.

One emission line is selected to constrain each elemental abundance. In Table 1, a question mark is appended to those lines with unreliable intensities (Table 3): the intensity of $\mathrm{Mg} \mathrm{I}] \lambda 4571+62$ is given as an upper limit as the lines are barely detected (TI05) and suspected to be blended with a WR feature (Guseva et al. 2000); detection of $\mathrm{Al}$ III $\lambda 1855$ is an estimate from a tracing of the HST spectrum; Si III] $\lambda 1883+92$ is barely seen in the IUE spectrum and only the first component of the doublet is detected in the HST spectrum (IT99). The abundances of $\mathrm{Mg}, \mathrm{Al}$ and $\mathrm{Si}$ are arbitrarily linked to that of argon (Table 1), assuming abundance ratios close to solar (Lodders 2003). For simplicity, the solar S/Ar ratio is also adopted and the computed sulfur line intensities can be used to scale $\mathrm{S} / \mathrm{H}$ according to any 
preferred criterion (Sect. 6.7). He I emission lines are blended with strong stellar absorption lines (ICF99). $\mathrm{He} / \mathrm{H}$ is set at 0.08 by number.

In the lower part of Table 1 we give observational constraints for the structural parameters of the shell, depending on assumptions. In preliminary constant-density "runs" $(N 0, N 1$, not genuine models; Sect. 5.1), a generalization of the approach of SS99 (Sect. 2.1) is adopted. A one-component model (M1; Sect. 5.2) shows the influence of Eq. (1). Two-component models (M2, M3 and $M 4$; Sect. 5.3) generalize $M 1$ according to Sect. 4.2.

\section{Results}

Input and output model properties are listed in the first column of Table 2 as: (1) five primary ionizing source parameters (Sect. 4.1); (2) resulting numbers of photons $\left(\mathrm{s}^{-1}\right) Q$ and $Q_{\mathrm{He}}$ emitted by the source above 13.6 and $24.6 \mathrm{eV}$ respectively; (3) four $(N 0, N 1, M 1)$ to six $(M 2, M 3, M 4)$ shell parameters (Sect. 4.2); (4) elemental abundances; (5) photon fraction $Q_{\mathrm{abs}} / Q$ absorbed in the shell; (6) mass $M_{\mathrm{gas}}$ of ionized gas in units of $10^{6} M_{\odot}$; (7) mean ionic fractions of $\mathrm{H}^{+}$and oxygen ions weighted by $N_{\mathrm{e}} ;(8)$ average $T_{\mathrm{e}}$ and $t^{2}$ weighted by $N_{\mathrm{e}} \times N_{\text {ion }}$ and average $N_{\mathrm{e}}$ weighted by $N_{\text {ion }}$ for $\mathrm{H}^{+}$and oxygen ions.

The model SEDs (Sect. 4.1) are shown in Fig. 1: panel (a) is common to $N 0, N 1$ and $M 1$; panels (b), (c) and (d) correspond to $M 2, M 3$ and $M 4$ respectively. Radiation is harder and stronger in panel (a) (see "hardness coefficient" $\alpha$ in caption to Fig. 1). The gas pressure laws $P(\tau)$, drawn in Fig. 2 (parameters in Table 2), illustrate the contrast between preliminary runs and adopted models.

Line identifications and observed de-reddened intensities are provided in Cols. 1 and 2 of Table 3. Computed intensities appear in Cols. 3 and 5 for Run $N 0$ and Model M2 respectively. Predictions are given for some unobserved lines (intensities are 10 times the quoted values for H I $1215 \AA$ and $2 h v$ ). The ratios of computed to observed intensities, noted " $N 0 / \mathrm{O}$ " and " $M 2 / \mathrm{O}$ ", appear in Cols. 4 and 6 for $N 0$ and $M 2$ respectively. Ideally, these ratios should be 1.00 for all observed lines.

Inasmuch as the convergence is completed, at least all lines used as model constraints (Table 1) must be exactly matched by construction (Table 3). To evaluate the models, these lines are therefore useless. Similarly, "redundent" lines (H I and He I series, etc.), which carry no astrophysically significant information in this context, as well as unobserved lines, can be discarded. Remaining "useful" lines are listed in Cols. 1, 2 of Table 4 and model intensities divided by observed intensities in Cols. 3-8 for $N 0-N 1, M 1-M 4$ respectively. These intensities are "predictions" in that they are not considered at any step of the convergence. In Table $4^{4}$, [O III] $] 4363 \AA$ stands out as the strongest, most accurately measured optical line. $Q_{\mathrm{abs}} / Q$ is repeated in Table 4.

\subsection{Constant density runs with filling factor: NO, N1}

$N 0$ is a preliminary run (Col. 2 of Table 2) in which $N_{\mathrm{H}}$ is constant and $Q, Q_{\mathrm{He}}, R_{\mathrm{i}}$ and $R_{\mathrm{f}}$ are as in the description by $\mathrm{SS} 99$ (corrected for the larger $D$ ). The convergence process, involving $\mathrm{O} / \mathrm{H}, \mathrm{Ne} / \mathrm{H}$, etc. (Table 1), is more complete than the one performed by SS99, but the differences in procedures do not change the conclusions. If $N_{\mathrm{H}}$ is in principle derived from $r([\mathrm{~S} \mathrm{II}])$, the

4 Since $[\mathrm{O} \mathrm{III}] \lambda 5007+4959$ is exactly matched, the entry [O III] $\lambda 4363$ in Tables 4 and 5 is the "normalized $r([\mathrm{OIII}])$ ", i.e., the ratio of the computed $r([\mathrm{O}$ III $])$ to the observed $r([\mathrm{O} \mathrm{III}])$.
Table 2. IZw 18 NW: model parameters and properties.

\begin{tabular}{|c|c|c|c|c|c|}
\hline \multirow{3}{*}{$\begin{array}{l}\text { Parameters } \\
\text { of model }\end{array}$} & \multirow{3}{*}{$\begin{array}{c}\text { Run }^{a} \\
\text { N0-N1 } \\
2-3\end{array}$} & \multicolumn{4}{|c|}{ Model $^{b}$} \\
\hline & & M1 & M2 & M3 & M4 \\
\hline & & 4 & 5 & 6 & 7 \\
\hline \multicolumn{6}{|c|}{ Central source parameters } \\
\hline$\overline{T 1 / 10^{4} \mathrm{~K}}$ & 10. & 10. & 8. & 8. & 12. \\
\hline$L 1 / 10^{41} \mathrm{erg} \mathrm{s}^{-1}$ & $3.5-1.6$ & 3.5 & 2.0 & 1.6 & 1.25 \\
\hline$\delta_{4}$ & .12-.67 & 0.73 & 0.83 & 0.93 & 0.24 \\
\hline$T 2 / 10^{4} \mathrm{~K}$ & 4. & 4. & 4. & 5. & 4. \\
\hline$L 2 / 10^{41} \mathrm{erg} \mathrm{s}^{-1}$ & $3.5-1.6$ & 3.5 & 2.0 & 1.6 & 2.5 \\
\hline $\log (Q)-51$ & $1.04-.71$ & 1.054 & 0.829 & 0.779 & 0.734 \\
\hline$-\log \left(Q_{\mathrm{He}} / Q\right)$ & $.462-.447$ & 0.445 & 0.523 & 0.493 & 0.539 \\
\hline \multicolumn{6}{|c|}{ Ionized shell parameters } \\
\hline $\bar{\epsilon}$ & $.0042-.31$ & 1.00 & 1.00 & 1.00 & 1.00 \\
\hline$P_{\text {in }} / k / 10^{5} \mathrm{CGS}$ & 38. -8.3 & 5.01 & 3.40 & 2.96 & 2.71 \\
\hline$P_{\text {out }} / k / 10^{5} \mathrm{CGS}$ & - & 23.2 & 21.7 & 25.4 & 26.8 \\
\hline$\tau_{\mathrm{c}}$ & - & 5.7 & 4.9 & 4.0 & 4.3 \\
\hline$f_{1}^{\text {cov }}$ & 1.- -0.43 & 0.20 & 0.26 & 0.23 & 0.29 \\
\hline$f_{2}^{\text {cov }}$ & - & - & 0.30 & 0.50 & 0.60 \\
\hline$\tau_{\mathrm{m}}$ or $\tau_{\mathrm{m}}(2)$ & $.96-300$. & 270. & 1.21 & 1.46 & 1.00 \\
\hline \multicolumn{6}{|c|}{ Elemental abundances by number $\left(\mathrm{H}=10^{7}\right)$} \\
\hline$\overline{\mathrm{C}}$ & $60-52$ & 45.8 & 38.8 & 35.3 & 47.4 \\
\hline $\mathrm{N}$ & $8.1-5.3$ & 3.9 & 4.1 & 4.0 & 3.8 \\
\hline $\mathrm{O}$ & $198-192$ & 172. & 168. & 162. & 173. \\
\hline $\mathrm{Ne}$ & $33-30$ & 26.4 & 25.7 & 24.8 & 26.9 \\
\hline $\mathrm{S}$ (Table 1) & $3.4-3.9$ & 5.0 & 4.3 & 4.3 & 5.0 \\
\hline $\mathrm{Ar}$ & $.77-.90$ & 1.14 & 0.99 & 0.98 & 1.13 \\
\hline $\mathrm{Fe}$ & $4.8-5.8$ & 5.8 & 6.1 & 6.0 & 6.5 \\
\hline \multicolumn{6}{|c|}{ Mean shell properties weighted by $N_{\mathrm{e}} \times N_{\text {ion }}$, except for $N_{\mathrm{e}}$} \\
\hline$\overline{Q_{\text {abs }} / Q}$ & $.20-.43$ & .200 & .343 & .380 & .426 \\
\hline$M_{\mathrm{gas}} / 10^{6} M_{\odot}$ & $.15-.92$ & 1.02 & 1.52 & 1.74 & 1.79 \\
\hline $\mathrm{H}^{+} / \mathrm{H}$ & $.998-.98$ & .957 & .961 & .963 & .948 \\
\hline $\mathrm{O}^{0} / \mathrm{O}$ & $.00-.017$ & .041 & .038 & .037 & .052 \\
\hline $\mathrm{O}^{+} / \mathrm{O}$ & $.076-.10$ & .122 & .125 & .129 & .131 \\
\hline $\mathrm{O}^{2+} / \mathrm{O}$ & $.910-.85$ & .784 & .793 & .790 & .773 \\
\hline $\mathrm{O}^{3+} / \mathrm{O}$ & $.014-.03$ & .049 & .043 & .043 & .042 \\
\hline$T_{\mathrm{e}}\left(\mathrm{H}^{+}\right) / 10^{4} \mathrm{~K}$ & $1.65-1.73$ & 1.873 & 1.859 & 1.896 & 1.839 \\
\hline$T_{\mathrm{e}}\left(\mathrm{O}^{0}\right) / 10^{4} \mathrm{~K}$ & $1.61-1.14$ & 1.040 & 1.013 & 1.012 & 1.003 \\
\hline$T_{\mathrm{e}}\left(\mathrm{O}^{+}\right) / 10^{4} \mathrm{~K}$ & $1.63-1.43$ & 1.320 & 1.315 & 1.309 & 1.272 \\
\hline$T_{\mathrm{e}}\left(\mathrm{O}^{2+}\right) / 10^{4} \mathrm{~K}$ & $1.66-1.75$ & 1.911 & 1.915 & 1.961 & 1.898 \\
\hline$T_{\mathrm{e}}\left(\mathrm{O}^{3+}\right) / 10^{4} \mathrm{~K}$ & $1.76-2.1$ & 2.576 & 2.402 & 2.449 & 2.479 \\
\hline$N_{\mathrm{e}}\left(\mathrm{H}^{+}\right) / \mathrm{cm}^{-3}$ & 99-18 & 17.3 & 11.1 & 9.8 & 9.4 \\
\hline$N_{\mathrm{e}}\left(\mathrm{O}^{0}\right) / \mathrm{cm}^{-3}$ & $99-8.5$ & 46.7 & 34.0 & 37.8 & 41.1 \\
\hline$N_{\mathrm{e}}\left(\mathrm{O}^{+}\right) / \mathrm{cm}^{-3}$ & $99-16$ & 62.7 & 48.0 & 54.0 & 54.8 \\
\hline$N_{\mathrm{e}}\left(\mathrm{O}^{2+}\right) / \mathrm{cm}^{-3}$ & $99-18$ & 15.4 & 10.2 & 8.9 & 8.5 \\
\hline$N_{\mathrm{e}}\left(\mathrm{O}^{3+}\right) / \mathrm{cm}^{-3}$ & 100-19 & 10.1 & 7.4 & 6.3 & 5.7 \\
\hline$t^{2}\left(\mathrm{H}^{+}\right)$ & $.002-.014$ & .032 & .026 & .028 & .030 \\
\hline$t^{2}\left(\mathrm{O}^{0}\right)$ & $.001-.018$ & .022 & .024 & .023 & .020 \\
\hline$t^{2}\left(\mathrm{O}^{+}\right)$ & $.0013-.02$ & .021 & .024 & .024 & .023 \\
\hline$t^{2}\left(\mathrm{O}^{2+}\right)$ & $.002-.008$ & .013 & .010 & .010 & .010 \\
\hline$t^{2}\left(\mathrm{O}^{3+}\right)$ & $.0010-05$ & .0008 & .0006 & .0007 & .0016 \\
\hline
\end{tabular}

${ }^{a}$ Constant $N_{\mathrm{H}} . N 0: N_{\mathrm{H}}=92 \mathrm{~cm}^{-3} ; N 1: N_{\mathrm{H}}=17.0 \mathrm{~cm}^{-3}$.

${ }^{b} N_{\mathrm{H}}$ from thermal pressure of Eq. (1).

sensitivity of $r([\mathrm{~S}$ II $])$ to $N_{\mathrm{e}}$ is relatively weak at the low density prevailing in the shell, while the exact value adopted for $N_{\mathrm{H}}$ may, in this particular structure, strongly influence the computed spectrum. By coherently changing $N_{\mathrm{H}}, \epsilon$ and $f^{\text {cov }}$, the three constraints $I(\mathrm{H} \beta)$, [O III]/[O II] and $R_{\mathrm{f}}$ can be fulfilled along a sequence. $N 0$ is extracted from this sequence by assuming, as in the SS99 run, a covering factor $f^{\text {cov }}=1$. The solution is close to the one chosen by SS99, with $N_{\mathrm{H}}=92 \mathrm{~cm}^{-3}, \epsilon=0.0042$, (radial) $\tau \sim 1\left(\equiv \tau_{\mathrm{m}}(2)\right.$ in Table 2$)$ and $r([\mathrm{~S} \mathrm{II}])$ only $-2.1 \%$ off the observed value. NO (Cols. 3, 4 of Table 3; Col. 3 of 

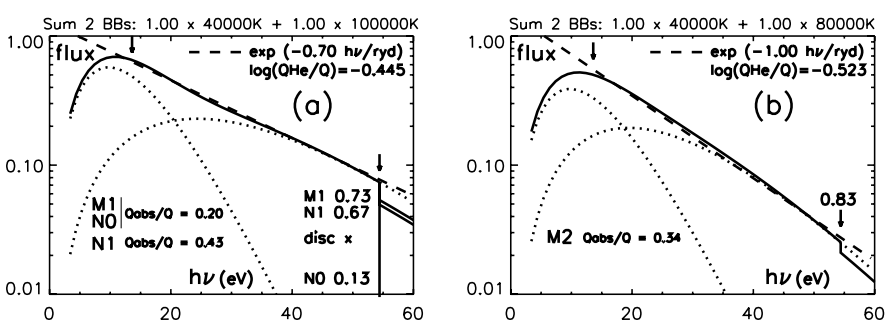

1.00 Sum 2 BBs: $1.00 \times 50000 \mathrm{~K}+1.00 \times 80000 \mathrm{~K}$
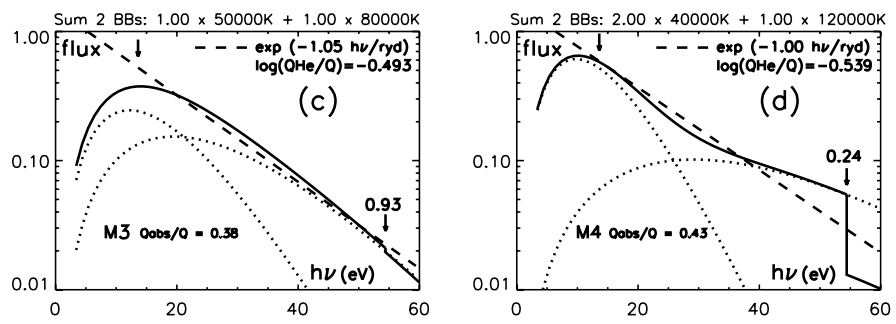

Fig. 1. Spectral energy distribution (SED) for I Zw $18 \mathrm{NW}$ models. Flux in $\mathrm{erg} \mathrm{s}^{-1} \mathrm{eV}^{-1}$ multiplied by an arbitrary constant versus photon energy $h v$ in eV. The SED (solid line) is the sum of two black bodies (dotted lines), with a discontinuity at 4 ryd (Sect. 4.1). Panel a) is common to $N 0, N 1$ and $M 1$. Panels b), c) and d) correspond to $M 2, M 3$ and $M 4$ respectively. Vertical arrows appear at 1 and 4 ryd. Straight dashed lines of the form $\exp (-\alpha h v / \mathrm{ryd})$ are exponential approximations of the SEDs over the range 1-4 ryd. The exponential expressions are written in the panels corresponding to the SEDs ( $\alpha=0.70,1.00,1.05$ and 1.00 in panels a) to d), with $\alpha$ a measure of the EUV continuum hardness). Some of the SED data provided in Table $2\left(Q_{\mathrm{He}} / Q\right.$, discontinuity factor $\delta_{4}$ and $\left.Q_{\mathrm{abs}} / Q\right)$ are recalled in the panels.

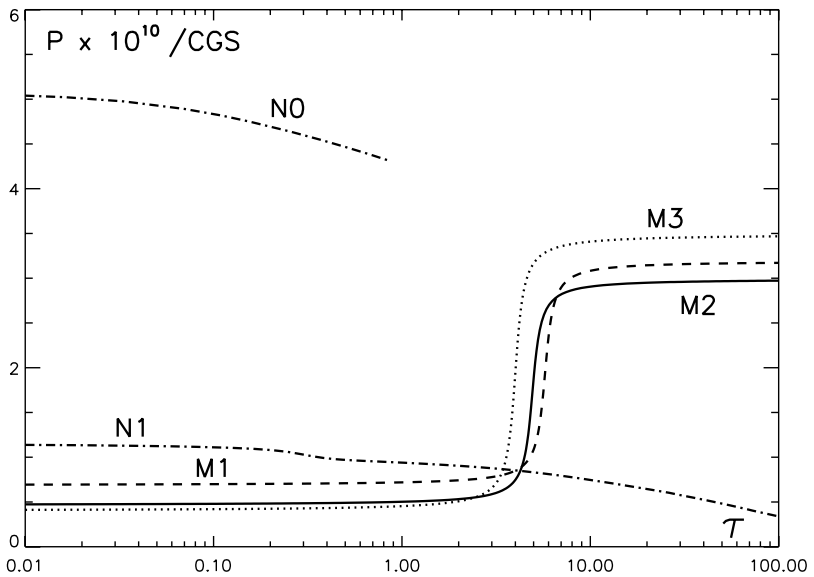

Fig. 2. Gas pressure versus optical depth $\tau$ for models $M 1$ (dashed line), $M 2$ (solid line) and $M 3$ (dotted line), governed by Eq. (1) with parameters in Table 2. $M 4$, close to $M 3$, is not shown. For comparison, the dash-dotted lines correspond to the constant density runs $N 0$ and $N 1$.

Table 4) confirms the problems met in SS99 with [O III] 44363 and $[\mathrm{OI}] \lambda 6300$ (Sect. 2.1).

$N 0$ also fails in that $Q_{\mathrm{abs}} / Q$ is half the expected value. Decreasing $Q$ (SED luminosity) implies to decrease $N_{\mathrm{H}}$ (for [O III $] /[\mathrm{O} \mathrm{II}]$ ) and increase $\epsilon$ (for $I(\mathrm{H} \beta)$ ). Decreasing $N_{\mathrm{H}}$ should help increase $T_{\mathrm{e}}$, thus $r([\mathrm{O} \mathrm{III}])$, and the high ionization lines, largely underestimated in $\mathrm{NO}$. Correlatively, [O III]/[O II] is restored for a larger $\tau_{\mathrm{m}}$, which helps increase [O $\left.\mathrm{I}\right]$ and other low-ionization lines. By further decreasing $N_{\mathrm{H}}$ and increasing $\epsilon$, whilst fine-tuning $f^{\text {cov }}$ to keep the outer shell radius $R_{\mathrm{f}}$ (and $I(\mathrm{H} \beta)$ ) and $\delta_{4}$ (for He II), it is possible to further increase $\tau_{\mathrm{m}}$ until the shell becomes radiation bounded. The resulting (unique) solution is $N 1$ (Col. 3 of Table 2; Col. 4 of Table 4), which
Table 3. Model outputs for IZw $18 \mathrm{NW}$ : NO and $M 2$.

\begin{tabular}{|c|c|c|c|c|c|}
\hline Line id./Models & Obs. & N0 & $\overline{\mathrm{N} 0 / \mathrm{O}}$ & $\overline{\mathrm{M} 2}$ & $\overline{\mathrm{M} 2 / \mathrm{O}}$ \\
\hline \multicolumn{6}{|c|}{ Absolute fluxes $\left(I(\mathrm{H} \beta)\right.$ in $\left.10^{-14} \mathrm{erg} \mathrm{cm}^{-2} \mathrm{~s}^{-1}\right)$} \\
\hline$\overline{I(\mathrm{H} \beta)}$ & 5.6 & 5.6 & 1.00 & 5.6 & 1.00 \\
\hline $1.43 \mathrm{GHz} / \mathrm{mJy}$ & 0.433 & 0.240 & 0.55 & 0.256 & 0.59 \\
\hline $8.45 \mathrm{GHz} / \mathrm{mJy}$ & 0.286 & 0.202 & 0.71 & 0.216 & 0.76 \\
\hline \multicolumn{6}{|c|}{ Relative line fluxes (wavelengths in $\AA$ or $\mu \mathrm{m}$ ) } \\
\hline H I 4861 & 1000. & 1000. & 1.00 & 1000. & 1.00 \\
\hline Н I 6563 & 2860. & 2840. & 0.99 & 2830. & 0.99 \\
\hline H I 4340 & 461. & 473. & 1.03 & 473. & 1.03 \\
\hline Н г 4102 & 266. & 267. & 1.00 & 267. & 1.00 \\
\hline НІ 1215 (/10) & - & 2950. & - & 3010. & - \\
\hline Н I $2 h v(/ 10)$ & - & 1550. & - & 1620. & - \\
\hline He I 3888 & 90.4 & 89.6 & 0.99 & 90.5 & 1.00 \\
\hline He I 4471 & 21.4 & 34.9 & 1.63 & 35.2 & 1.64 \\
\hline Не I 5876 & 67.7 & 92.0 & 1.36 & 91.3 & 1.35 \\
\hline He I 6678 & 25.3 & 25.3 & 1.00 & 25.6 & 1.01 \\
\hline He I 7065 & 24.4 & 23.4 & 0.96 & 22.9 & 0.94 \\
\hline He I 10830 & - & 251. & - & 190. & - \\
\hline He II 4686 & 36.8 & 36.8 & 1.00 & 36.8 & 1.00 \\
\hline C III] 1909+07 & 467. & 467. & 1.00 & 467. & 1.00 \\
\hline Si III] $1882+92$ & 270.: & 229. & 0.85 & 340. & 1.26 \\
\hline Al III $1855+63$ & 111.: & 42.9 & 0.39 & 82.9 & 0.75 \\
\hline O III] 1664 & $<230$ & 127. & $>.5$ & 208. & $>.9$ \\
\hline C IV 1549 & 510.: & 74.2 & 0.14 & 334. & 0.65 \\
\hline Si IV 1397$\}^{a}$ & $<300$ & 22.7 & $>.1$ & 127. & $>.5$ \\
\hline O IV] 1398$\}$ & $*$ & 2.0 & $*$ & 24.9 & $*$ \\
\hline [N II] 6584+48 & 9.2 & 9.2 & 1.00 & 9.2 & 1.00 \\
\hline [O I ] 6300+63 & 8.5 & 0.12 & 0.01 & 8.6 & 1.01 \\
\hline [O II] 3726+29 & 238. & 238. & 1.00 & 238. & 1.00 \\
\hline$[\mathrm{O}$ II $] 7320+30$ & 6.3: & 9.5 & 1.50 & 7.5 & 1.18 \\
\hline [O III $] 5007+.$. & 2683. & 2680. & 1.00 & 2680. & 1.00 \\
\hline [O III] 4363 & 65.9 & 47.8 & 0.73 & 63.2 & 0.96 \\
\hline [O III $] 51.8 \mu \mathrm{m}$ & - & 174. & - & 137. & - \\
\hline [O III] $88.3 \mu \mathrm{m}$ & - & 216. & - & 213. & - \\
\hline [O IV] $25.9 \mu \mathrm{m}$ & 49.1 & 18.2 & 0.37 & 47.8 & 0.97 \\
\hline [Ne II] $12.8 \mu \mathrm{m}$ & 9.0: & 1.3 & 0.14 & 1.9 & 0.21 \\
\hline [Ne III] 3868+.. & 191. & 191. & 1.00 & 191. & 1.00 \\
\hline [Ne III] $15.5 \mu \mathrm{m}$ & 45.7 & 60.0 & 1.31 & 48.9 & 1.07 \\
\hline $\mathrm{Mg} \mathrm{I}] 4571+62$ & $<3.0$ & 1.5 & $>.5$ & 1.2 & $>.4$ \\
\hline [Si II $] 34.8 \mu \mathrm{m}$ & 157. & 4.7 & 0.03 & 22.0 & 0.14 \\
\hline [S II] $6716^{\prime}$ & 22.5 & 6.7 & 0.30 & 17.6 & 0.78 \\
\hline [S II] 6731 & 16.9 & 5.1 & 0.30 & 13.1 & 0.78 \\
\hline [S II] 4068$\}$ & 3.7 & 1.1 & 0.41 & 2.2 & 0.99 \\
\hline$[\mathrm{Fe} \mathrm{v}] 4071\}$ & * & 0.4 & $*$ & 1.5 & * \\
\hline [S III $] 9531+.$. & 114. & 130. & 1.15 & 113. & 0.99 \\
\hline [S III] 6312 & 6.7 & 6.0 & 0.89 & 5.7 & 0.85 \\
\hline [S III] $18.7 \mu \mathrm{m}$ & 28.0 & 32.2 & 1.15 & 26.2 & 0.95 \\
\hline [S III $] 33.5 \mu \mathrm{m}$ & 120. & 54.5 & 0.45 & 48.0 & 0.40 \\
\hline [S IV] $10.5 \mu \mathrm{m}$ & 48.0 & 41.7 & 0.87 & 92.6 & 1.93 \\
\hline [Ar III] 7136+.. & 23.5 & 23.5 & 1.00 & 23.5 & 1.00 \\
\hline [Ar III] $8.99 \mu \mathrm{m}$ & - & 8.6 & - & 8.1 & - \\
\hline [Ar IV] 4711\} & 8.6 & 1.5 & 0.76 & 8.2 & 1.53 \\
\hline He I 4713$\}$ & $*$ & 5.0 & $*$ & 5.0 & $*$ \\
\hline [Ar IV] 4740 & 4.5 & 1.2 & 0.26 & 6.2 & 1.39 \\
\hline$[\mathrm{Fe} \mathrm{II}] 5.34 \mu \mathrm{m}$ & - & 0.1 & - & 10.8 & - \\
\hline$[\mathrm{Fe} \mathrm{II}] 26.0 \mu \mathrm{m}$ & 34. & 0.0 & 0.00 & 3.4 & 0.10 \\
\hline [Fe III $] 4658$ & 4.5 & 4.5 & 1.00 & 4.5 & 1.00 \\
\hline [Fe III $] 4986$ & 7.4 & 5.8 & 0.78 & 7.0 & 0.94 \\
\hline [Fe III] $22.9 \mu \mathrm{m}$ & - & 2.2 & - & 3.2 & - \\
\hline [Fe IV] 4906 & 3.0: & 1.6 & 0.54 & 3.1 & 1.02 \\
\hline$[\mathrm{Fe} \mathrm{v}] 4227$ & 1.8 & 1.5 & 0.84 & 5.5 & 3.10 \\
\hline
\end{tabular}

${ }^{a}$ In Col. 1, blends are indicated by braces. The observed intensity of a blend is attributed to the first line and an asterisk to the second line.

improves upon $N 0$ concerning [O I] and high-ionization lines, while $Q_{\mathrm{abs}} / Q \equiv f^{\text {cov }}=0.43$ is slightly too large. Because of the 
Table 4. Model "predictions" for I Zw 18 NW: model intensities divided by observed intensities.

\begin{tabular}{|c|c|c|c|c|c|c|}
\hline Line ident. & $\begin{array}{c}\text { Obs. } \\
2\end{array}$ & $\begin{array}{c}\mathrm{N} 0-\mathrm{N} 1 \\
3-4\end{array}$ & $\begin{array}{c}\text { M1 } \\
5\end{array}$ & $\begin{array}{c}\text { M2 } \\
6\end{array}$ & $\begin{array}{c}\text { M3 } \\
7\end{array}$ & $\begin{array}{c}\text { M4 } \\
8\end{array}$ \\
\hline$\overline{Q_{\mathrm{abs}}} / Q^{a}$ & 0.37 & $.20-.43$ & 0.20 & 0.34 & 0.38 & 0.43 \\
\hline CIV 1549 & 500: & $.14-.41$ & 1.12 & 0.65 & 0.64 & 1.08 \\
\hline$[\mathrm{O}$ & 8.5 & .01-.59 & 1.19 & 1.01 & 0.95 & 1.38 \\
\hline [O II] 7320+ & 6.3: & $1.5-1.24$ & 1.19 & 1.18 & 1.19 & 1.15 \\
\hline [O III] 4363 & 65.9 & $.73-.82$ & 0.96 & 0.96 & 1.00 & 0.94 \\
\hline [O IV] $25.9 \mu$ & 49.1 & $.37-.84$ & 1.08 & 0.97 & 0.94 & 0.98 \\
\hline [Ne II] $12.8 \mu$ & 9.0: & $.14-.10$ & 0.14 & 0.21 & 0.22 & 0.23 \\
\hline$[\mathrm{Ne}$ III $] 15.5 \mu$ & 45.7 & $1.31-1.22$ & 1.09 & 1.07 & 1.02 & 1.11 \\
\hline [Si II] $34.8 \mu$ & 157. & $.03-.10$ & 0.17 & 0.14 & 0.14 & 0.18 \\
\hline Si III] $1882+$ & 270: & $.85-.99$ & 1.12 & 1.26 & 1.34 & 1.16 \\
\hline 16 & 22.5 & $.30-.54$ & 0.96 & 0.78 & 0.77 & 1.10 \\
\hline 31 & 16.9 & $.30-.51$ & 0.96 & 0.78 & 0.77 & 1.10 \\
\hline I] $4068^{b}$ & $3.7^{b}$ & $.30-.43$ & 0.73 & 0.58 & 0.58 & 0.80 \\
\hline [S III] 9531+ & 114. & $1.15-1.05$ & 0.95 & 0.99 & 1.00 & 0.92 \\
\hline 12 & 6.7 & .89- & 0.77 & 0.85 & 0.87 & 0.75 \\
\hline I] $18.7 \mu$ & 28. & $1.15-1.01$ & 0.94 & 0.95 & 0.94 & 0.91 \\
\hline I] $33.5 \mu$ & 120. & 年-.44 & 0.39 & 0.40 & 0.40 & 0.39 \\
\hline & 48. & $.87-1.50$ & 2.15 & 1.93 & 1.97 & 2.15 \\
\hline [Ar IV] 4740 & 4.5 & $.26-.73$ & 1.87 & 1.39 & 1.47 & 1.76 \\
\hline$[\mathrm{Fe}$ II] $26.0 \mu$ & 34. & 年-.05 & 0.10 & 0.10 & 0.10 & 0.14 \\
\hline [Fe III] 4986 & 7.4 & $.78-1.15$ & 0.90 & 0.94 & 0.89 & 0.89 \\
\hline [Fe IV] 4906 & 3.0 & $.54-.70$ & 0.96 & 1.02 & 1.09 & 1.02 \\
\hline$[\mathrm{Fe} \mathrm{V}] 4227$ & 1.8: & $.84-2.3$ & 2.1 & 3.1 & 3.2 & 3.1 \\
\hline
\end{tabular}

${ }^{a} Q_{\text {abs }} / Q$ : absolute values.

${ }^{b} \lambda 4068$ intensity not corrected for $[\mathrm{Fe} \mathrm{v}] \lambda 4071$ (Table 3 ).

much lower density, $N_{\mathrm{H}}=17 \mathrm{~cm}^{-3}$, the ratio $r([\mathrm{~S} \mathrm{II}])$ is now $+4.7 \%$ off, worse than in $N 0$, yet not decisively inacceptable. Nevertheless, the normalized $r([\mathrm{O} \mathrm{III}])$, enhanced from 0.73 to 0.82 , is still significantly too small. This failure of $N 1$ is illustrated in the upper panels of Figs. 3-5. The runs of $N_{\mathrm{e}}$ and $T_{\mathrm{e}}$ with nebular radius $R$ are shown in Figs. 3a, 4a for $N 0$ and Figs. 3b, $4 \mathrm{~b}$ for $N 1$. Ionic fractions of oxygen versus $\tau$ are shown in Figs. $5 \mathrm{a}$ and $5 \mathrm{~b}$ for $N 0$ and $N 1$ respectively. In $N 0, T_{\mathrm{e}}$ is above $1.55 \times 10^{4} \mathrm{~K}$ everywhere. In $N 1$, the inner $T_{\mathrm{e}}$ is $3800 \mathrm{~K}$ higher than in $N 0$, but $\mathrm{O}^{2+}$ is abundant up to $\tau=15$, where $T_{\mathrm{e}}$ is below $1.40 \times 10^{4} \mathrm{~K}$ and the average $T_{\mathrm{e}}([\mathrm{O} \mathrm{III}])$ is not much increased.

This generalization shows that no solution with constant $N_{\mathrm{H}}$ exists even for the rather hard SED adopted by SS99. In an extreme variant of $N 1$, the SED is just one $10^{5} \mathrm{~K}$ black body (converged $\left.L=2.7 \times 10^{41} \mathrm{erg} \mathrm{s}^{-1}, \delta_{4}=0.41, \log \left(Q_{\mathrm{He}} / Q\right)=-0.27\right)$, but the normalized $r([\mathrm{O}$ III $])=0.87$ is still too small, despite the unrealistically hard SED.

\subsection{A one-sector photoionization model: $M 1$}

Model $M 1$ includes the same primary source as Run $N 0$ and again only one sector, but with $N_{\mathrm{H}}$ controlled by Eq. (1) (Fig. 2). Parameters appear in Col. 4 of Table 2 and predictions in Col. 5 of Table 4. The lines [O III] $\lambda 4363$ and [O I] 6300 are improved compared to Run $N 0$ (and even N1), as are [S II] and [O IV].

The decisive merit of Model $M 1$ is to demonstrate that, with no extra free parameter, no change of shell size and no significant change of source SED, the " $r([\mathrm{O} \mathrm{III}])$ problem" met in $N 0$ can be solved by considering radiation-bounded filaments embedded in a lower density (higher ionization) medium instead of a clumped shell at constant density. While the normalized $r([\mathrm{O}$ III $]$ ) is 0.96 ([O III] in Table 4), no dense or distant clumps of the kind postulated by SS99 (Sect. 2) are needed to account for low-ionization lines. The pressure contrast is $<5$.
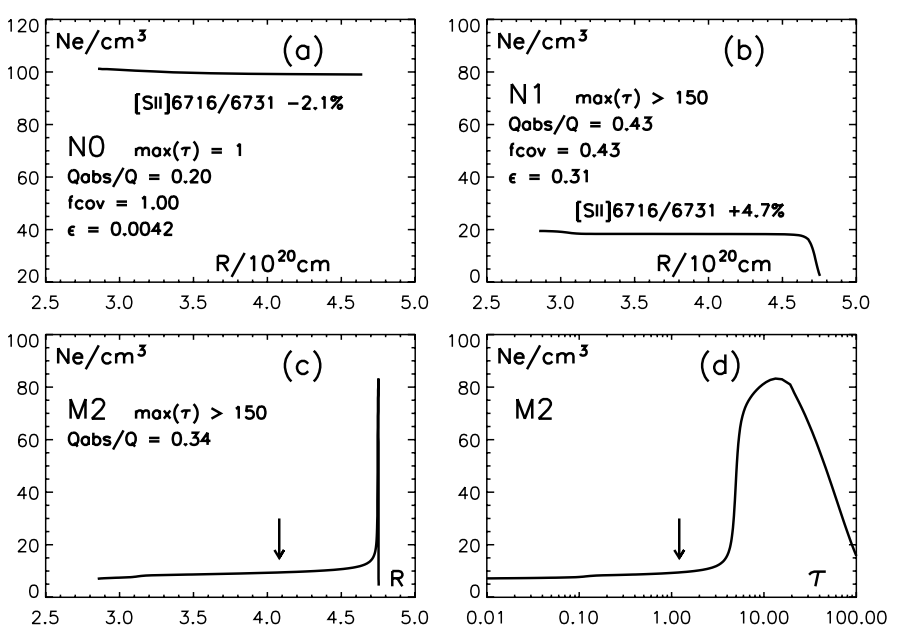

Fig. 3. Local electron density $N_{\mathrm{e}}$ versus shell radius $R$ in IZw $18 \mathrm{NW}$ models: a) $N 0$, b) $N 1$, c) $M 2$; and versus optical depth $\tau$ at 1 ryd: d) $M 2$. In panels c) and d), the vertical arrows indicate the boundary of the matter-bounded sector 2 of Model $M 2$. Also provided are the optical thickness " $\max (\tau)$ " ("> $>150$ " means "radiation bounded"), the fraction of primary photons absorbed $Q_{\mathrm{abs}} / Q$, and, where relevant ( $N 0$ and $\left.N 1\right)$, the covering factor $f^{\text {cov }}$, the filling factor $\epsilon$ and the departure of the computed [S II] doublet ratio from observation in $\%$.
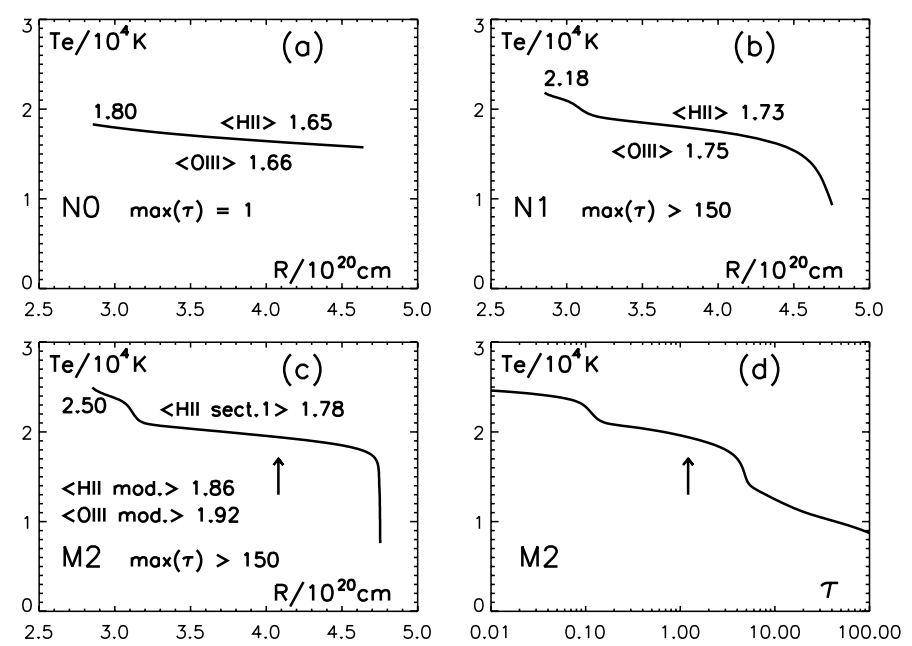

Fig. 4. As in Fig. 3 for local electron temperature $T_{\mathrm{e}} / 10^{4} \mathrm{~K}$. The maximum $T_{\mathrm{e}}$ and average $T_{\mathrm{e}}$ 's weighted by $N_{\mathrm{e}} \times \mathrm{N}\left(\mathrm{H}^{+}\right)$and by $N_{\mathrm{e}} \times \mathrm{N}\left(\mathrm{O}^{2+}\right)$ are noted along each curve. In panel $\mathbf{c}),\left\langle T_{\mathrm{e}}\right\rangle$ is given for both the radiation bounded sector $(\langle$ sect. 1$\rangle)$ and the full model $M 2(\langle\bmod \rangle$.$) .$

$Q_{\text {abs }} / Q$ is again too small, but $Q$ cannot decrease because $\delta_{4}$ is close to unity (Table 2). In $N 0, \delta_{4}$ was (perhaps anomalously) small, enabling a shift from $N 0$ to $N 1$. Hardening the already hard primary ( $\alpha=0.7$ due to large $T 1$; Fig. 1$)$ would enhance [Ar IV], predicted too strong. Also, $f^{\text {cov }}$ is only 0.20 , resulting in an artificial cigar-like radial distribution, in which the low-density gas exactly shields the denser filaments from direct primary radiation.

Obviously, the limits of the one-sector model are being reached. A matter-bounded sector is to be added for the sake of a larger absorbed fraction of photons in the shell, but not principally to improve the already quite satisfactory intensities of [O III] $\lambda 4363$ and $[\mathrm{O}$ I $] \lambda 6300$. 

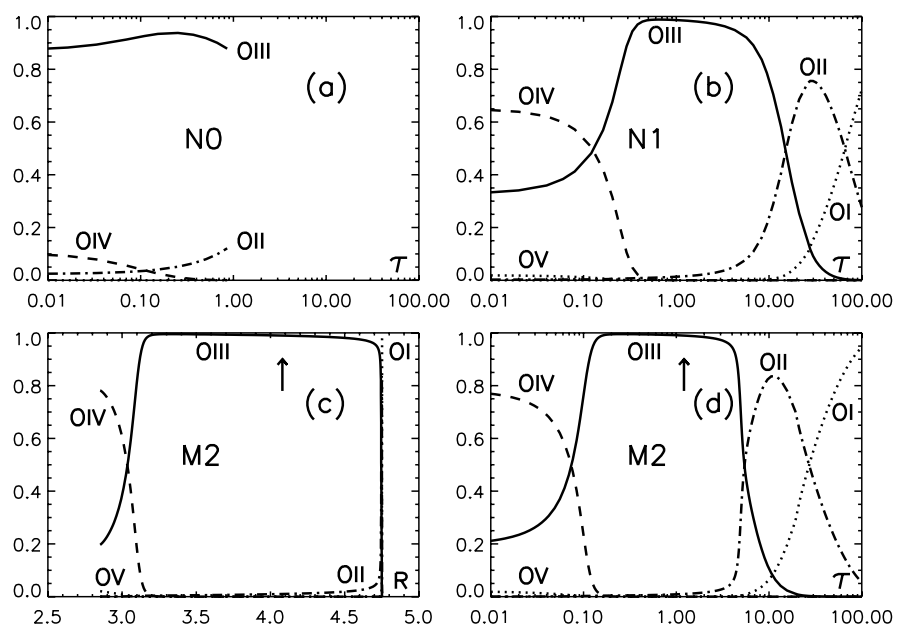

Fig. 5. Local fractional concentrations of $\mathrm{O}^{0}$ (dotted line), $\mathrm{O}^{+}$(dotdashed line), $\mathrm{O}^{2+}$ (solid line), $\mathrm{O}^{3+}$ (dashed line) and $\mathrm{O}^{4+}$ (dotted line again) versus $\tau$ in I Zw $18 \mathrm{NW}$ models: a) $N 0$, b) $N 1$, d) $M 2$; and versus $R$ : c) $M 2$. Vertical arrows as in Fig. 3.

\subsection{Two-sector photoionization models: M2, M3, M4}

The enhancement of He II and [O III] related to the matterbounded sector must be balanced by a weaker/softer SED. Models illustrate the influence of the SED (Fig. 1).

Given a SED and both covering factors, then $\tau_{\mathrm{m}}(2), \tau_{\mathrm{c}}$ and $\delta_{4}$ can be fine-tuned to account for $\mathrm{I}(\mathrm{H} \beta),[\mathrm{O} \mathrm{III}] /[\mathrm{O}$ II $]$ and $\mathrm{He}$ II. Iterations along the same lines as for Model $M 1$ eventually lead to a model, provided that the limits on parameters are respected (Table 1). A two-parameter model sequence can be attached to any SED by considering several pairs $\left(f_{1}^{\text {cov }}, f_{2}^{\text {cov }}\right)$, but little freedom is attached to $f_{1}^{\text {cov }}$, as Sector 1 is where $\sim 75 \%$ of $\mathrm{H} \beta$ and most of [O II] come from. Then $f_{1}^{\text {cov }}$ must be of the order of, or moderately larger than the $f^{\mathrm{cov}}$ of the one-sector model, say, in the range $0.2-0.3$. Also $f_{2}^{\text {cov }}$ cannot be small since one-sector models are rejected (Sect. 5.2) and $f_{1}^{\text {cov }}+f_{2}^{\text {cov }}$ must be kept significantly less than unity to enable He II excitation beyond the shell (Sect. 4.2), implying $f_{2}^{\text {cov }} \sim 0.3-0.6$.

\subsubsection{Model M2 and variants}

Model M2 (Col. 5 of Table 2) is the first "complete" model. $Q_{\mathrm{abs}} / Q$ is at the low end of the nominal interval. Ouput of line intensities (Cols. 5-6 of Table 3; Col. 6 of Table 4) is to be contrasted to the $N 0$ output. Runs of $N_{\mathrm{e}}$ and $T_{\mathrm{e}}$ with $R$ are shown in Figs. 3c, 4c. The sharp "spike" of the $N_{\mathrm{e}}$ curve shows how thin a radiation-bounded filament is compared to the shell. Runs of $N_{\mathrm{e}}$ and $T_{\mathrm{e}}$ are best seen in plots versus $\tau$ (Figs. $3 \mathrm{~d}, 4 \mathrm{~d}$ ). In plots for $M 2$, vertical arrows mark the outer boundary of Sector 2 . Ion fractions $\mathrm{O}^{n+} / \mathrm{O}$ versus $R$ and $\tau$ are shown in Figs. 5c,d.

Sufficient ionization is maintained in $M 2$ due to the lower average density, which also helps increase $T_{\mathrm{e}}$ in the high-ionization layers, despite the significantly softer radiation field (smaller $Q_{\mathrm{He}} / Q$ and larger $\alpha$, Fig. $1 \mathrm{~b}$ ): the inner $T_{\mathrm{e}}$ is now $2.5 \times 10^{4} \mathrm{~K}$. In Fig. $4 \mathrm{~d}$, the jumps of $T_{\mathrm{e}}$ at $\tau \sim 0.1$ and $\sim 4.7$ correspond to the boundaries of the $\mathrm{He}^{2+}$ shell (fairly well traced by $\mathrm{O}^{3+}$ in Fig. 5d) and the filament respectively. Comparing Fig. 5d to Fig. 5b, the ionic fractions are qualitatively similar in Model M2 and Run $N 1$, but the transition from $\mathrm{O}^{2+}$ to $\mathrm{O}^{+}$is sharper and occurs at a smaller optical depth in $M 2$.

Average properties and abundances of Model M2 are similar to those of $M 1$ and the predicted [O III] $\lambda 4363$ intensity is again
Table 5. Influence of shell parameters on selected line intensity predictions for models $M 2$ and $M 4$.

\begin{tabular}{l|cccccc}
\hline \hline Param./line & M2 & M2b & M2c & M4b & M4 & M4c \\
& 2 & 3 & 4 & 5 & 6 & 7 \\
\hline$f_{1}^{\text {cov }}$ & 0.26 & 0.26 & 0.22 & 0.29 & 0.29 & 0.22 \\
$f_{2}^{\text {cov }}$ & 0.30 & 0.60 & 0.60 & 0.30 & 0.60 & 0.60 \\
$\tau_{\mathrm{m}}(2)$ & 1.21 & 0.62 & 0.92 & 2.17 & 1.01 & 1.57 \\
$\tau_{\mathrm{c}}$ & 4.9 & 4.7 & 3.8 & 4.6 & 4.3 & 3.0 \\
$P_{\text {in }} / k / 10^{5}$ & 3.4 & 3.4 & 3.1 & 2.7 & 2.7 & 2.4 \\
$P_{\text {out }} / k / 10^{5}$ & 21.7 & 22.5 & 24.6 & 26.8 & 26.8 & 26.8 \\
$\mathrm{O} / \mathrm{H} \times 10^{7}$ & 168. & 165. & 162. & 178. & 173. & 171. \\
\hline$[\mathrm{O} \mathrm{I}] 6300+$ & 1.01 & 1.00 & 0.92 & 1.42 & 1.37 & 1.06 \\
{$[\mathrm{O} \mathrm{III}] 4363$} & 0.96 & 0.97 & 1.00 & 0.92 & 0.94 & 0.95 \\
{$[\mathrm{O} \mathrm{IV}] 25.9 \mu$} & 0.97 & 0.91 & 0.92 & 1.05 & 0.98 & 0.99 \\
{$[\mathrm{~S} \mathrm{II}] 6716$} & 0.78 & 0.81 & 0.79 & 1.05 & 1.10 & 0.84 \\
{$[\mathrm{~S} \mathrm{IV}] 10.5 \mu$} & 1.93 & 1.97 & 2.05 & 2.07 & 2.15 & 1.92 \\
{$[$ Ar IV $] 4740$} & 1.39 & 1.48 & 1.62 & 1.55 & 1.77 & 1.81 \\
\hline
\end{tabular}

slightly weak, although the score of $M 2$ is significantly better for [Ar IV] and [S IV] (Col. 6 of Table 4).

Variants to Model $M 2$ can be obtained by changing $f_{1}^{\text {cov }}$ and $f_{2}^{\text {cov }}$ within limits, while retaining source parameters (except for minute fine-tuning of $\delta_{4}$ ). In Col. 1 of Table 5 are listed 7 shell parameters and 6 lines extracted from Table 4. M2 (Col. 2 of Table 5) is compared to models $M 2 b$ (Col. 3) and $M 2 c$ (Col. 4). Increasing $f_{2}^{\text {cov }}$ from a small to a large value, with $f_{1}^{\text {cov }}$ left unchanged, structure parameters are not much changed except for a decrease of $\tau_{\mathrm{m}}(2)$ and a small decrease of $\mathrm{O} / \mathrm{H}$ due to the larger weight of the hot high-ionization zone. Accordingly, [Ar IV] is increased, but [O III] $\lambda 4363$ is increased by only $1 \%$. Decreasing $f_{1}^{\text {cov }}$ from 0.26 to $0.22, \mathrm{H} \beta$ is recovered by increasing $\tau_{\mathrm{m}}(2)$ and [O II] $\lambda 3727$ by decreasing $\tau_{\mathrm{c}}$, with the consequence that $P_{\text {in }}$ must decrease, thus $T_{\mathrm{e}}$ increase and $\mathrm{O} / \mathrm{H}$ decrease. The $\lambda 4363$ intensity increases up to the observed value, [Ar IV] $\lambda 4740$ and [S IV] $\lambda 10.5 \mu$ increase and [O I] $\lambda 6300$ decreases.

\subsubsection{Models $M 3$ and $M 4$}

In Model $M 3, T 2 / 10^{4} \mathrm{~K}$ is enhanced from 4 to $5 . Q_{\mathrm{abs}} / Q$ is larger than in $M 2$ due to lower luminosity. The larger $T 2$ increases the average energy of photons absorbed in the $\mathrm{O}^{2+}$ region and the intensity of [O III] $\lambda 4363$ is slightly larger. In the selected example, [O III] is again exactly matched as in $M 2 c$, but for more "standard" $f_{1}^{\text {cov }}$ and $f_{2}^{\text {cov }}$ (Col. 6 of Table 2). Line intensity predictions are slightly improved (Col. 7 of Table 4 versus Col. 4 of Table 5).

In Model $M 4, T 2$ is again as in $M 2$, while $T 1 / 10^{4} \mathrm{~K}$ is enhanced from 8 to 12 and $L 1 / L 2$ is halved. $Q_{\mathrm{abs}} / Q$ is close to its allowed maximum due to radiation hardening, which also leads to $\delta_{4} \ll 1$ (Col. 7 of Table 2). The large flux just below 4 ryd enhances simultaneously the high and low ionization lines, but the heating of the $\mathrm{O}^{2+}$ region is lesser and the large $T 1$ (positive curvature of the SED) does not favour a large $r$ ([O III]) (Col. 8 of Table 4). From Table 5, variants $M 4 b$ (Col. 5) and $M 4 c$ (Col. 7) of M4 (Col. 6) fail to enhance [O III] $\lambda 4363$ up to the observed value. Increasing the source luminosity by $20 \%$, thus decreasing $Q_{\text {abs }} / Q$ from 0.43 to 0.36 , has no effect on the predicted [O III] after convergence. 


\section{Discussion}

Irrespective of the "technical" demand raised by $Q_{\mathrm{abs}} / Q$ in Sect. 5.2, a two-sector model is the minimum complexity of any shell topology (Sect. 4.2). The two degrees of freedom attached to the matter-bounded sector are inescapable.

Model M4 (and variants) appears slightly less successful than $M 2$ and $M 3$ concerning [O III] $\lambda 4363$ and the highionization lines ([Ar IV] $\lambda 4740$, [S IV] $\lambda \lambda 10.5 \mu) . M 4$ has a less likely SED and presents the largest $P_{\text {out }} / P_{\text {in }}$. The discussion focuses on Models $M 2$ and $M 3$, with $M 2$ the "standard" from which variants are built.

\subsection{Spectral energy distribution}

Accounting for a $Q_{\text {abs }} / Q$ larger than, say, $1 / 3$ turns out to be demanding. Selected models correspond to nearly maximum possible values for each SED. Acceptable $Q_{\text {abs }} / Q$ can indeed be obtained, but the latitude on the SED and power of the ionizing source is narrow. The uncertainties in evolutionary synthetic cluster models (Appendix C) and in the evolutionnary status of I Zw $18 \mathrm{NW}$ itself are sufficient to provisionally accept the "empirical" SED corresponding to preferred models $M 2$ or $M 3$ (Fig. 1) as plausible. The "predicted" typical trend is $L_{v}(h v=$ $1 \rightarrow 4$ ryd $) \propto \exp (-h v /$ ryd $)$.

\subsection{Ionized gas distribution}

The model is most specific in that emission lines partly arise from a low density gas, while the largest $N_{\mathrm{e}}$ is $N_{\mathrm{e}}$ ([S II $\left.]\right)$. A density $\leq 10 \mathrm{~cm}^{-3}$ (Table 2) appears very low by current standards of photoionization models for BCDs (Appendix A.1). Nevertheless, the superbubble model of Martin (1996) is consistent with a current $S F R=0.02 M_{\odot} \mathrm{yr}^{-1}$ for the whole $\mathrm{NW}+\mathrm{SE}$ complex. The two best estimates in the compilation by $\mathrm{Wu} 07$ are 0.03 and $0.02 M_{\odot} \mathrm{yr}^{-1}$. Adopting half the Martin (1996) rate and a wind injection radius of $0.1 \mathrm{kpc}\left(1.5^{\prime \prime}\right.$ at $13 \mathrm{Mpc}$ ) for the NW cluster alone, expression (10) in Veilleux et al. (2005) suggests an inner pressure of the coronal gas $P / k \sim 3 \times 10^{5} \mathrm{~K} \mathrm{~cm}^{-3}$, hence an ambient ISM number density $\sim 12 \mathrm{~cm}^{-3}$ in the inner region $\left(T_{\mathrm{e}} \sim 2.5 \times 10^{4} \mathrm{~K}\right.$; Sect. 5), or else $N_{\mathrm{H}} \sim 12 / 2.3=5 \mathrm{~cm}^{-3}$ for a photoionized gas in pressure balance with the coronal phase which presumably permeats the shell. Although $\epsilon$ is unity in models, this phase can fill in the volume corresponding to Sector 3 .

\subsection{Optical and UV lines}

[O III] lines are discussed in Sects. 6.7 and 6.8. C IV and Si III] are accounted for within uncertainties. Other UV lines are elusive (Col. 6 of Table 3$)$.

Computed fluxes for 8 optical lines are within $20 \%$ of observation (Table 4), which is satisfactory considering the weakness of some of the lines. The 10-15\% discrepancy on the ratio [S III] 6312/9531 does not challenge the model itself, given the various uncertainties. The $\sim 20 \%$ underestimation of [S II] should be considered with respect to [S III]. The line $\lambda 9531$ is matched, but the far-red flux may be less reliable, and $\lambda 6312$ departs from observations about in the same way as [S II], but $\lambda 6312$ is a weak line. The exact status of [S II] is undecided.

The $N_{\mathrm{e}}$-sensitive intensity ratio [Fe III] $\lambda 4986 / \lambda 4658$ is somewhat small in $N 0$, large in $N 1$ and more nearly correct in $\mathrm{Mi}$ models. Would [S II] be emitted in a high- $N_{\mathrm{e}}$ gas component as suggested by SS99 and V02, then [Fe III] 4986, roughly co-extensive with [O II], would be undetectable. The weak line [Fe IV] 4906, co-extensive with [O III], confirms the ion distribution of the $M i$ models and the iron abundance, although the agreement with observation is partly fortuitous. [Fe v] 4227 is overpredicted by a factor $\sim 3$, but the observed intensity is very uncertain and could be 2-3 times stronger than the quoted value, as judged from published tracing (TI05). Also, only one computation of collision strengths has ever been done for the optical lines of the difficult [Fe V] ion (Appendix D.3). Finally, the ionic fraction $\mathrm{Fe}^{4+} / \mathrm{Fe}$, less than $5 \%$, is subject to ionization balance inaccuracy. The predicted intensity $\sim I(\lambda 4227 \AA) / 4$ of [Fe V] 4071 enhances the computed flux of [S II] 4068 up to the observed value (Tables 3 and 4 ).

The observed He I line intensities are inconsistent (Table 3), due to stellar lines (Sect. 4.3). [Ar IV] 4711, blended with He I 4713, is therefore useless. The weak [Ar IV] 4740 tends to be overestimated by $\sim 50 \%$ in the preferred models. Trial calculations show that, adopting a recombination coefficient 12 times the radiative one (instead of 8 times, Appendix D.3) and dividing $\mathrm{Ar} / \mathrm{H}$ by 1.13 , [Ar III] and [Ar IV] would be matched in $M 2$.

\subsection{Infrared fine-structure lines}

\subsection{1. [NeIII] and [SIII]}

The reliably observed IR lines with optical counterparts, [Ne III] $15.5 \mu$ and [S III] $18.7 \mu$, are very well matched, confirming the scaling adopted for the SPITZER fluxes and the model temperatures. The $M i$ models, globally hotter, are more successful than the $N i$ runs. No $t^{2}$ in excess of the one of the adopted configuration (Fig. 8d) is required.

The predicted intensity of [S III] $33.5 \mu$ is only $40 \%$ of the observed value. Since the theoretical ratio of the [S III] IR lines is insensitive to conditions in I Zw 18, looking for alternative models is hopeless. The collision strengths $\Omega$ for the [S III] lines may not be of ultimate accuracy, as the results of Tayal \& Gupta (1999) and Galavis et al. (1995) differ, but the more recent $\Omega$ are likely more accurate. Also, the predicted [S III] $33.5 \mu$ is even worse using older data. Since Wu07 cast doubt on the accuracy of the flux calibration at the end of the SPITZER spectrum, it is assumed that the $[\mathrm{S} \mathrm{III]}$ atomic data are accurate and that the observed fluxes around $\lambda 34 \mu$ should be divided by 2.3 .

\subsection{2. [OIV]}

If the drift of flux calibration at $\lambda 34 \mu$ (Sect. 6.4.1) smoothly vanishes towards shorter wavelengths, the [O IV] $26 \mu$ flux may still be overestimated. Conversely, the SPITZER field of view encompasses I Zw $18 \mathrm{SE}$, which emits little He II, leading to underestimate $[\mathrm{O} I V] / \mathrm{H} \beta$ in I Zw $18 \mathrm{NW}$. Since these effects act in opposite directions, the original $[\mathrm{O} \mathrm{IV}] / \mathrm{H} \beta$ is adopted for $\mathrm{IZw} 18 \mathrm{NW}$.

As shown in Fig. 5, $\mathrm{O}^{3+}$ and $\mathrm{O}^{2+}$ coexist in the $\mathrm{He}^{2+}$ zone. $\mathrm{O}^{3+} / \mathrm{O}^{2+}$ and therefore [O IV] $\lambda 25.88 \mu / \mathrm{He}$ II $\lambda 4686$ as well are sensitive to $N_{\mathrm{e}}$. In $N 0$, He II is matched and [O IV] is strongly underpredicted (Table 4). The predicted [O IV] flux improves in the conditions of $N 1$ and even by-pass observation in $M 1$, whose ionizing flux is however too large (Sect. 5.2). In the standard Model M2, the predicted [OIV] exactly matches observation after adding the blended line [Fe V] $25.91 \mu$, whose computed flux is $\sim 2 \%$ of [O IV]. Since relevant atomic data are reliable, [O IV] $25.88 \mu$ indicates that $N_{\mathrm{e}}$ must be of the order of $10 \mathrm{~cm}^{-3}$ in the He II emitting region of the I Zw $18 \mathrm{NW}$ shell. The model density results from general assumptions (photoionization by stars, shell geometry, $\epsilon=1$, Eq. (1), etc.) and a requirement 
to match a few basic line intensities with no reference to highionization lines, but He II. The computed [O IV] intensity is a true prediction, especially as the models were essentially worked out prior to IR observations: the spectrum presented by $\mathrm{Wu}$ et al. (2006) showed the predicted [O IV] line, finally noted by Wu07.

\subsection{3. [SIV]}

The predicted [S IV] $10.5 \mu$ flux is twice the observed one. The collision strengths obtained by Tayal (2000) and Saraph \& Storey (1999) for this line are in good agreement. The average fractional concentration of $\mathrm{S}^{3+}, \sim 1 / 3$, is stable in different models because sulfur is mostly distributed among the three ions $\mathrm{S}^{2+}-\mathrm{S}^{4+}$. Displacing the ionization balance by changing, e.g., the gas density tends to make either $\mathrm{S}^{2+}$ or $\mathrm{S}^{4+}$ migrate to $\mathrm{S}^{3+}$. Only in the unsatisfactory run $N O$ is $[\mathrm{S}$ IV] accounted for.

Two-sector, constant-pressure "models" allowing $\epsilon<1$ and using the SED of Model $M 2$ were run with the conditions $Q_{\text {abs }} / Q>0.3$ and $\mathrm{O} / \mathrm{H}<1.7 \times 10^{-5}$. In these trials, the [O III 5007 and $r([\mathrm{~S} \mathrm{II}])$ constraints (Table 1) are relaxed and the observed [S IV]/[S III] ratio is exactly matched varying $N_{\mathrm{e}}$ (gas pressure) and $\epsilon$. Despite ample freedom and because of the higher $N_{\mathrm{e}} \sim 25 \mathrm{~cm}^{-3}$, the computed [O IV] flux is at most $60 \%$ of the observed one. Thus, forgetting other difficulties, the suggestion is that the excess [S IV] flux can only be cured at the expense of [O IV]. A broader exploration of the SED (discontinuities) and the gas distribution could be undertaken.

The [S IV] $10.5 \mu$ flux published by Wu et al. (2006) was $25 \%$ larger than according to $\mathrm{Wu} 07$. The new value should be preferred, but this difference is at least indicative of possible uncertainties. The ratio $[\mathrm{S} \mathrm{IV}] / \mathrm{H} \beta$ may also be intrinsically larger in $\mathrm{IZw} 18 \mathrm{NW}$ than in I Zw $18 \mathrm{SE}$.

The theoretical ionization balance of some ions of sulfur (and argon) is subject to uncertainties (Appendix D.3). The observed [S IV]/[S III] ratio can be recovered in $M 2$ if the $\mathrm{S}^{2+}$ recombination coefficient is multiplied by a factor of 2.3 , which is perhaps acceptable (Badnell 2007, private communication): then, both [S III] and [S IV] are matched if $\mathrm{S} / \mathrm{H}$ is divided by 1.33 , with the caveat that the predicted [S II] intensities are divided by 1.3. A combination of observational and theoretical effects just listed could alleviate the "[S IV] problem".

\subsection{Low ionization fine-structure lines}

Although the error bars of order $10 \%$ quoted by $\mathrm{Wu} 07$ may not include all sources of uncertainties, both [Ne II] $12.78 \mu$ and $[\mathrm{Fe} \mathrm{II}] 25.98 \mu$ are detected in high-resolution mode. [Si II] $34.80 \mu$ is strong, even though the flux quoted by Wu07 (Table 4) may be too large (Sect. 6.4.1). Usually, most of [Si II] $35 \mu$ and [Fe II] $26 \mu$ arise from a Photon-Dominated Region (PDR), at the warm HI interface between an ionization front and a molecular cloud (e.g., Kaufman et al. 2006). Schematically in a PDR, the photo-electric heating by UV radiation on dust grains (and other molecular processes) is balanced by fine-structure (and molecular) line emission. The small reddening intrinsic to IZw 18 (Sect. 3.2) and the "large" gaseous iron content (Sect. 6.7) imply that little dust is available. Molecules and PAHs are not detected in IZw 18 (Vidal-Madjar et al. 2000; Leroy et al. 2007; Wu07). The classical PDR concept may therefore not apply to I Zw 18 , raising the question of the origin of [Si II] $35 \mu$ and [Fe II] $26 \mu$, both underpredicted by factors of 5-10 in the models (Table 4).

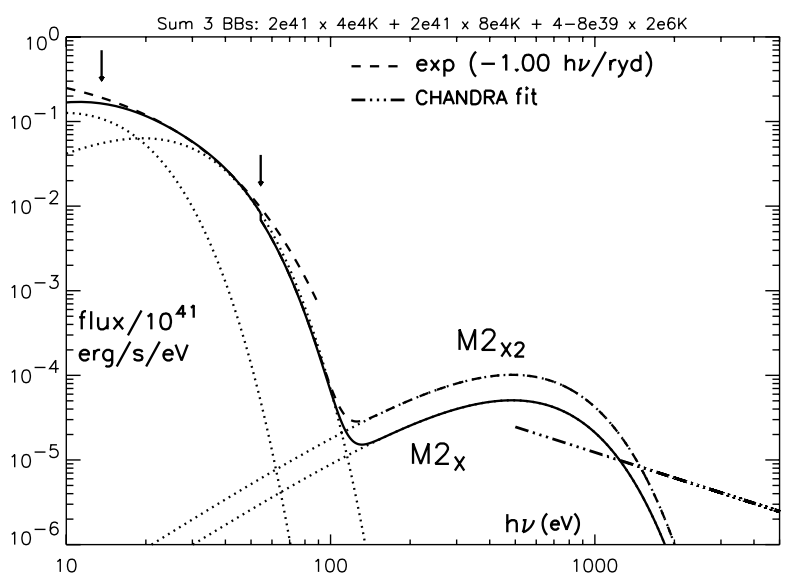

Fig. 6. As in Fig. $1 \mathrm{~b}$ for variants of $M 2$ comprising a soft X-ray emitting black body: Model $M 2_{\mathrm{X}}$ (solid line) and $M 2_{\mathrm{X} 2}$ (dash-dotted line). Ordinate unit flux is now $10^{41} \mathrm{erg} \mathrm{s}^{-1} \mathrm{eV}^{-1}$. Dash-triple-dotted line: one possible fit to the CHANDRA data for IZw $18 \mathrm{NW}$.
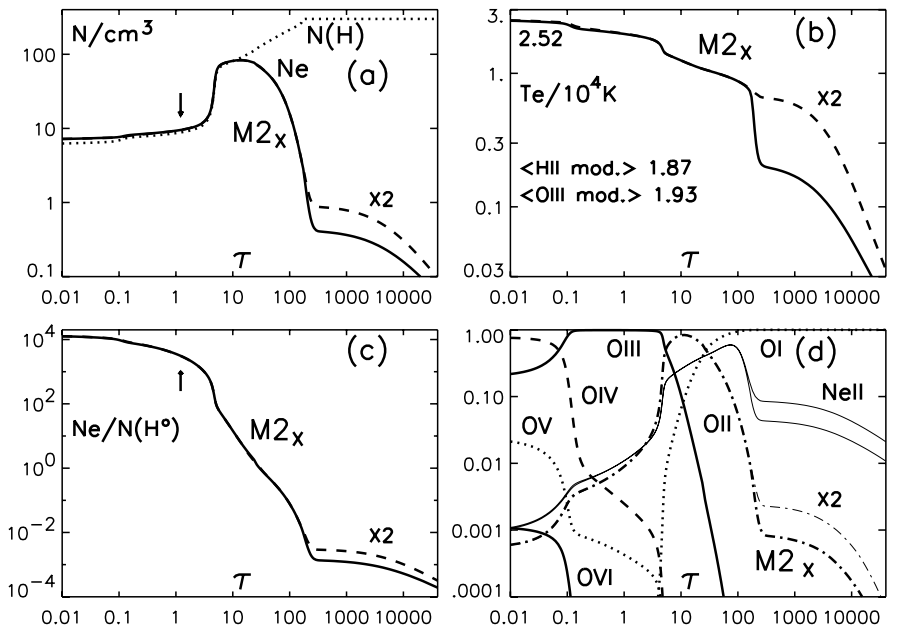

Fig. 7. Model $M 2_{\mathrm{X}}$ for IZw $18 \mathrm{NW}$. a) Electron density $N_{\mathrm{e}}$ (solid line) and total hydrogen density $N(\mathrm{H})$ (dotted line) versus optical depth $\tau$ at 1 ryd: $N(\mathrm{H})$ levels out at $300 \mathrm{~cm}^{-3}$. b) Electron temperature $T_{\mathrm{e}} / 10^{4} \mathrm{~K}$ : the maximum value and average values weighted by $N_{\mathrm{e}} \times N\left(\mathrm{H}^{+}\right)$and by $N_{\mathrm{e}} \times N\left(\mathrm{O}^{2+}\right)$ are noted for the full 2-sector model $(\langle\bmod \rangle$.$) . c) Ratio of$ $N_{\mathrm{e}}$ to atomic hydrogen density $N\left(\mathrm{H}^{0}\right)$. In panels a)-c), the dashed lines correspond to $M 2_{\mathrm{X} 2}$. d) Local fractional concentrations of $\mathrm{O}^{0}$ (dotted line), $\mathrm{O}^{+}$(dot-dashed line), $\mathrm{O}^{2+}$ (solid line), $\mathrm{O}^{3+}$ (dashed line), $\mathrm{O}^{4+}$ (dotted line again) and $\mathrm{O}^{5+}$ (solid line again): the thinner dash-dotted line $(\tau>200)$ corresponds to $\mathrm{O}^{+}$in $M 2 \mathrm{X} 2$ and the thinner solid line (split into $M 2_{\mathrm{X}}$ and $M 2_{\mathrm{X} 2}$ for $\tau>200$ ) to $\mathrm{Ne}^{+}$.

A way to produce a "pseudo-PDR" is X-ray heating. Two new variants of $M 2$ are considered, in which a hot black body representing a soft X-ray emission from IZw $18 \mathrm{NW}$ is added to the original SED. The adopted temperature is $T_{\mathrm{X}}=2 \times 10^{6} \mathrm{~K}$ and the luminosity $L_{\mathrm{X}}=4$ and $8 \times 10^{39} \mathrm{erg} \mathrm{s}^{-1}$ for variants $M 2_{\mathrm{X}}$ and $M 2_{\mathrm{X} 2}$ respectively (Fig. 6). The actual X-ray luminosity of IZw $18, \sim 1.6 \times 10^{39} \mathrm{erg} \mathrm{s}^{-1}$ in the $0.5-10 \mathrm{kev}$ range of CHANDRA mainly arises from the centre of IZw $18 \mathrm{NW}$ and is consistent with a power law of slope -1 (Thuan et al. 2004), drawn in Fig. 6. The SED for $M 2_{\mathrm{X}}$ is a relatively high, yet plausible extrapolation of the CHANDRA data. $M 2_{\mathrm{X} 2}$ is considered for comparison. $N_{\mathrm{H}}$, still governed by Eq. (1), levels out at $300 \mathrm{~cm}^{-3}$.

The run of physical conditions with $\tau$ is shown in Fig. 7 for $M 2_{\mathrm{X}}$. While $\left\langle T_{\mathrm{e}}\right\rangle$ is increased by only $\sim 100 \mathrm{~K}$ in the $\mathrm{H}$ II region, 
Table 6. Fine-structure line intensities ${ }^{a}$ and X-rays.

\begin{tabular}{lccccccc}
\hline \hline Line $^{b}$ & Obs. & \multicolumn{2}{c}{$M 2$} & \multicolumn{2}{c}{$M 2_{\mathrm{X}}$} & \multicolumn{2}{c}{$M 2_{\mathrm{X} 2}$} \\
\hline H I colls. $^{c}$ & - & no & yes & no & yes & no & yes \\
[C II] 158. & - & 2.1 & 2.1 & 124 & 119 & 224 & 204 \\
{$[\mathrm{OI}]$ 63.2 } & - & 4.7 & 4.6 & 274 & 210 & 624 & 450 \\
{$[\mathrm{Ne}$ II] 12.8 } & 9 & 1.9 & 3.6 & 1.9 & 5.5 & 2.0 & 16 \\
{$[\mathrm{Si}$ II 34.8 } & $157^{d}$ & 21 & 22 & 37 & 80 & 55 & 190 \\
{$[\mathrm{Ar}$ II] 7.0 } & - & 1.2 & 1.3 & 1.4 & 2.2 & 2.2 & 7.9 \\
{$[\mathrm{Fe}$ II] 26.0 } & 34 & 3.4 & 4.8 & 5.8 & 38 & 9.3 & 108 \\
{$[\mathrm{Fe}$ II] 35.3 } & - & 0.8 & 1.4 & 1.1 & 9.5 & 1.7 & 33 \\
\hline
\end{tabular}

${ }^{a}$ In units $\mathrm{H} \beta=1000 .{ }^{b}$ Wavelengths in $\mu \mathrm{m} .{ }^{c}$ Inelastic collisions of [Si II], [Fe II], [Ne II] and [Ar II] with H I omitted ("no") or included ("yes"). ${ }^{d}$ The re-calibrated [Si II] flux is 68 after Sect. 6.4.2.

a warm low-ionization layer develops beyond the ionization front. The computation is stopped at $T_{\mathrm{e}}=100 \mathrm{~K}\left(\tau \sim 7 \times 10^{4}\right.$, compared to 250 in $M 2$ ). In $M 2_{\mathrm{X} 2}$ (dashed lines in Figs. $7 \mathrm{a}-\mathrm{c}$ ), the new layer is hotter and more ionized (final $\tau \sim 1.2 \times 10^{5}$ ). The geometrical thickness of the HI layer is $21 \%$ and $35 \%$ of the HII shell in $M 2_{\mathrm{X}}$ and $M 2_{\mathrm{X} 2}$ respectively. In the HI zone, $\mathrm{O}^{+} / \mathrm{O}$ does not exceed $\sim 10^{-3}$, in marked contrast with $\mathrm{Ne}^{+} / \mathrm{Ne}$, overplotted as a thin solid line in Fig. 7d. Lines [Ne II] $12.8 \mu$ and [Ar II] 7.0 $\mu$ are usually discarded in PDR models on the basis that the ionization limits of $\mathrm{Ne}^{0}$ and $\mathrm{Ar}^{0}$ exceed 1 ryd. Here, owing to the scarcity of free electrons and the lack of charge exchange with $\mathrm{H}^{0}$, photoionization by soft X-rays can keep $1-10 \%$ of these elements ionized.

In H I regions, cooling is due to inelastic collisions with $\mathrm{H}^{0}$. Reliable collisional rates exist for the main coolents [C II] $157 \mu$ (Barinovs et al. 2005) and [O I] 63 $\mu$ (Abrahamsson et al. 2007; several processes need be considered for [OI]: see Chambaud et al. 1985; Péquignot 1990) and for [Si II] 35 $\mu$ (Barinovs et al. 2005), but not for, e.g., [Fe II] $26 \mu$. Following Kaufman et al. (2006), it is assumed that the cross-section for $\mathrm{H}^{0}+\mathrm{Si}^{+}$also applies to fine-structure transitions of other singly ionized species. Concerning [Fe II] (ground state ${ }^{6} \mathrm{D}_{9 / 2}$ ), collisions to ${ }^{6} \mathrm{D}_{7 / 2}$ follow the above rule, but cross-sections for transitions to the next ${ }^{6} \mathrm{D}_{J}$ are taken as $2 / 3,2 / 4$, etc. of the first one.

Low-ionization IR lines, including dominant coolents and other unobserved lines, are considered in Table 6. Line identifications and observed intensities appear in Cols. 1 and 2. The results of two computations are provided for $M 2$ (Cols. 3, 4), $M 2_{\mathrm{X}}$ (Cols. 5, 6) and $M 2_{\mathrm{X} 2}$ (Cols. 7, 8). In the first one, the excitations of [Si II], [Fe II], [Ne II], and [Ar II] (but not [O I] and [C II]) by collisions with $\mathrm{HI}$ are inhibited. In the second one, they are included according to the above prescription. Comparing different odd columns ("no") of Table 6, the rise of line intensities as the H I zone develops shows that the excitation of, e.g., [Si II] by free electrons is still active. [Ne II] is stable, due to the scarcity of $\mathrm{Ne}^{+}$ (Fig. 7d) relative to $\mathrm{Si}^{+}$. Comparing now odd columns to even columns, it is seen that H I collisions, ineffective in the "normal" $\mathrm{H}$ II region model $M 2$ (except, quite interestingly, for [Ne II]), strongly enhance the excitation rates. After subtracting emission from the $\mathrm{H}$ II region (M2), H I collisions contribute $75-80 \%$ of the excitation (virtually $100 \%$ in the case of [Ne II]).

Concerning [Si II] $35 \mu$, the atomic data are not controversial and the re-calibrated flux of 68 is reasonably well defined (Sect. 6.4.2). Then $M 2_{\mathrm{X} 2}$ (Col. 8 of Table 6) is excluded, while $M 2_{\mathrm{X}}$ (Col. 6) or an even weaker soft X-ray source (closer to the CHANDRA extrapolation) can account for [Si II]. Although the remarkably coherent predictions for [Si II] 35 $\mu$ and [Fe II] $26 \mu$ are partly fortuitous, they are consistent with (1) the [Fe II] collision strength being correctly guessed, (2) Fe/Si the same in the ionized and neutral gas, and (3) the excitation by soft X-ray heating is viable. Concerning [Ne II] $12.8 \mu$, the discrepancy with observation (factor 0.6) may not be significant, as the line is weak and its detection in the low-resolution mode is not taken as certain by Wu07. The collision strength may be too small.

Summarizing, a plausible extrapolation to soft X-rays of the CHANDRA flux can provide an explanation for the relatively high intensity of [Si II] $35 \mu$ and other fine-structure lines in I Zw 18. This is considerable support to the general picture of photoionization as the overwhelmingly dominant cause of heating of the $\mathrm{H}$ II region, since heating by conversion of mechanical energy appears unnecessary even in regions protected from ionization and heating by star radiation. Full confirmation should await reliable collisional excitation rates by $\mathrm{H}$ I for finestructure lines of all singly ionized species. The soft X-rays from I Zw $18 \mathrm{NW}$ have little effect on the $\mathrm{H}$ II region: both [O I] 6300 and [O IV] $25.9 \mu$ are increased by $3 \%$ and [Fe VI] by $30 \%$. The $1.7 \%$ enhancement of [O III] 4363 is of interest (Sect. 6.8).

\subsection{Stability of results}

The relative stability of the predicted line intensities is a consequence of the set of constraints (Table 1). Allowing for a range of values, a broader variety of results could be obtained. Are the conclusions dependent on input data?

The only basic line showing substantial variability in different spectroscopic studies is [O II] 3727. This line is sometimes found to be stronger than the adopted value (VI98; TI05). In a new variant $M 2 v$ of Model $M 2$, the observed [O II] intensity is assumed to be $20 \%$ larger than in Tables 3 and 4 and the covering factors are left unchanged. The inevitable $18 \%$ increase of the already too strong line [O II] $7320+30$ is not very significant, considering that the $\lambda 7325$ flux is very uncertain and may not correspond to a slit position with stronger $\lambda 3727$. Due to the larger fractional abundance of $\mathrm{O}^{+}, \mathrm{O} / \mathrm{H}$ is increased by $5 \%$. The greater weight of low-ionization layers induces a 3\% increase of $\mathrm{Ne} / \mathrm{H}$ and a $13 \%$ decrease of $\mathrm{N} / \mathrm{H}$ and $\mathrm{Fe} / \mathrm{H}$, as the $[\mathrm{N} \mathrm{II}]$ and [Fe III] intensities were left unchanged. Both [Ar IV] and [S IV] decrease by a few $\%$, whilst [O IV] increases by $6 \%$ and both [S II] and [O I] increase by $\sim 11 \%$. Ar/H and the [S III] lines increase by only $1 \%$ and [O III] $\lambda 4363$ is unchanged.

In a more extreme example, $M 2 c v_{\mathrm{X}}$, with an assumed [O II] intensity of 322 instead of 238 (factor 1.35), the $f \mathrm{~s}$ as in $M 2 c$ and the SED as in $M 2_{\mathrm{X}}$ (re-converged $\tau_{\mathrm{c}}=2.5$ and $\left.P_{\text {in }} / P_{\text {out }}=9\right)$, [O III] is exactly matched again, [O I ] is $+2 \%$ off, $[\mathrm{O}$ IV $]+6 \%$, [S IV] $+87 \%$ and $[\mathrm{S} \mathrm{II}]$ only $-8 \%$.

Thus, changes are moderate and alleviate difficulties noted in Sects. 6.3 and 6.4, e.g., the weakness of the [S II] doublet. The computed $r[\mathrm{O} \mathrm{III}]$ is robust.

\subsection{Elemental abundances}

To first order, $\mathrm{O} / \mathrm{H}$ reflects $\left\langle T_{\mathrm{e}}\left(\mathrm{O}^{2+}\right)\right\rangle$, related to $r([\mathrm{O} \mathrm{III}])$, i.e., the predicted [O III] $\lambda 4363$ intensity. Models $M 3$ and $M 2 c$ both almost exactly fit $\lambda 4363$ and share the same $\mathrm{O} / \mathrm{H}=1.62 \times 10^{-5}$, which is the best estimate, provided that (1) oxygen lines were given optimal observed intensities, (2) these models faithfully represent the $\mathrm{H}$ II region, and (3) collision strengths are accurate.

Concerning line intensities, [O III] 5007 is quite stable in different spectra of I Zw $18 \mathrm{NW}$ and the reasonably large, yet representative, ratio $r([\mathrm{O}$ III] $)$ (Sect. 3.3) is taken for granted, since our objective is deciding whether this specific ratio can be explained 

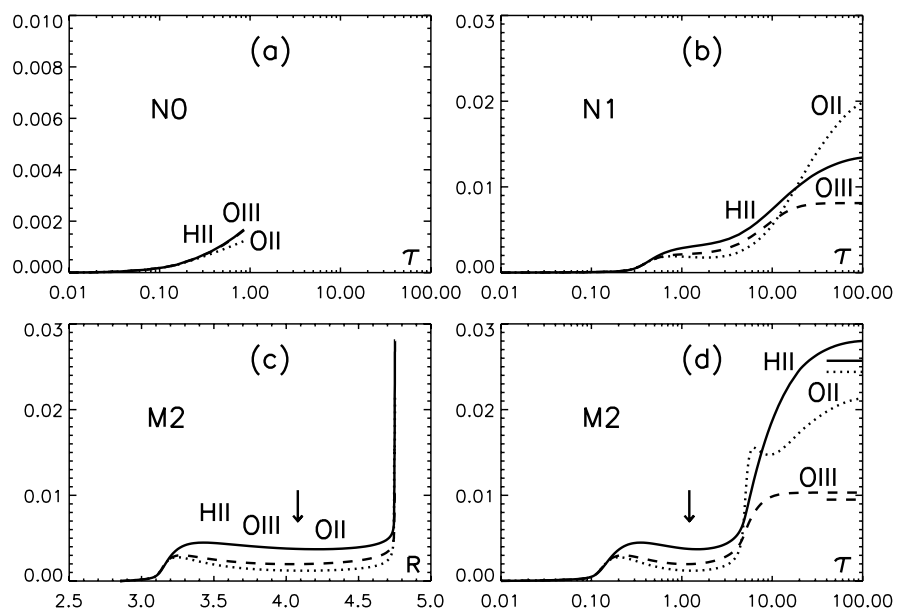

Fig. 8. Cumulative mean squared relative temperature fluctuations $t^{2}$ (Peimbert 1967) weighted by $N_{\mathrm{e}} \times N\left(\mathrm{H}^{+}\right)$(solid line), $N_{\mathrm{e}} \times N\left(\mathrm{O}^{+}\right)$(dotted line) and $N_{\mathrm{e}} \times N\left(\mathrm{O}^{2+}\right)$ (dashed line) versus $\tau$ in I Zw $18 \mathrm{NW}$ models: a) $N 0$, b) $N 1$, d) $M 2$; and versus $R$ : c) $M 2$. Note the expanded vertical scale in panel a). The short horizontal bars to the right of panel d) correspond to the means over the full model. Vertical arrows as in Fig. 3.

assuming photoionization by stars. In model $M 2 c v_{\mathrm{X}}$ (Sect. 6.6), which also fits the [O III] lines, [O II] 3727 was assumed to be enhanced by $35 \%$, leading to $\mathrm{O} / \mathrm{H}=1.74 \times 10^{-5}$.

Concerning models, the difference between the $T_{\mathrm{e}}$ directly derived from $r([\mathrm{OIII}]), T_{\mathrm{e}}([\mathrm{O} \mathrm{III}])=19850 \mathrm{~K}$, and the $N_{\mathrm{e}} \times$ $N\left(\mathrm{O}^{2+}\right)$ weighted average, $\left\langle T_{\mathrm{e}}\left(\mathrm{O}^{2+}\right)\right\rangle=19650 \mathrm{~K}$, corresponds to a formal $t^{2}([\mathrm{O} \mathrm{III}])=0.012$, similar to the computed $t^{2}\left(\mathrm{O}^{2+}\right)=$ 0.010 . This difference makes only a $1 \%$ difference for $\mathrm{O}^{2+} / \mathrm{H}^{+}$ and an empirical estimate neglecting $t^{2}$ should nearly coincide with model results for this ion. A major feature of the $T_{\mathrm{e}}$ profile is that the difference $\left\langle T_{\mathrm{e}}\left(\mathrm{O}^{2+}\right)\right\rangle-\left\langle T_{\mathrm{e}}\left(\mathrm{O}^{+}\right)\right\rangle$, which was only $300 \mathrm{~K}$ in $N 0$ and $3200 \mathrm{~K}$ in $N 1$, is $6600 \mathrm{~K}$ in the best models. The models are essential in providing a $T_{\mathrm{e}}$ to derive $\mathrm{O}^{+} / \mathrm{H}^{+}$from [O II] 3727 , as $T_{\mathrm{e}}$ ([O II $]$ ) is poorly determined from the uncertain [O II $] 7325$ and $t^{2}([\mathrm{O}$ II $])$ is inaccessible.

A "canonical" $\mathrm{O} / \mathrm{H}$ for I Zw $18 \mathrm{NW}$ is $1.46 \times 10^{-5}$ (SK93, ICF99), $11 \%$ less than the present $1.62 \times 10^{-5}$, out of which $4 \%$ are due to collisional excitation of $\mathrm{H} \beta$ and the remaining $7 \%$ could be a non-trivial consequence of the relatively large $t^{2}$ obtained for some ions in the present models (Table 2; Fig. 8), although differences in collision strengths may also intervene at the $2 \%$ level (Appendix D.2).

The silicon and sulfur abundances were not fine-tuned in models. The computed Si III] flux loosely suggests dividing the assumed $\mathrm{Si} / \mathrm{H}$ by 1.25 . Concerning $\mathrm{S} / \mathrm{H}$, [S II] is underestimated, [S III] globally underestimated and [S IV] overestimated. Correcting the ionization balance $[\mathrm{S} \mathrm{IV}] /[\mathrm{S} \mathrm{III}], \mathrm{S} / \mathrm{H}$ should be divided by 1.3 in the best models (Sect. 6.4.3), but [S II] is then underestimated. Since a combination of effects may explain the overestimation of $[\mathrm{S} \mathrm{IV}]$, the model $\mathrm{S} / \mathrm{H}$ is tentatively divided by 1.15. Similarly, [Ar IV]/[Ar III] is best accounted for if $\mathrm{Ar} / \mathrm{H}$ is divided by 1.13 (Sect. 6.3), but the [Ar IV] line is weak. The adopted correction factor is 1.06. Thus, S/Ar in IZw 18 is within $10 \%$ of the solar value, in agreement with the conclusion of Stevenson et al. (1993). The iron abundance relies on the [Fe III] lines, since [Fe II] $26 \mu$ does not arise from the $\mathrm{H}$ II region, while the $[\mathrm{Fe} \mathrm{IV}]$ and $[\mathrm{Fe} \mathrm{V}]$ intensities are uncertain. The [Fe III] intensities are from a spectrum in which [O II] 3727 is stronger than average (TI05). In variant $M 2 c v_{\mathrm{X}}$ (Sect. 6.6), where the intensity of [O II] is multiplied by 1.35 , both the
Table 7. Abundances in I Zw $18 \mathrm{NW}$ compared to solar and Galactic halo stars.

\begin{tabular}{l|c|cc|c|cc}
\hline \hline & & $\mathrm{X} / \mathrm{O}$ & $\mathrm{X} / \mathrm{O}$ & {$[\mathrm{X} / \mathrm{H}]$} & {$[\mathrm{X} / \mathrm{Fe}]$} & {$[\mathrm{X} / \mathrm{Fe}]$} \\
$\mathrm{El}$. & $M^{a}$ & $M$ & $\mathrm{IT}^{b}$ & $M$ & $M$ & $\mathrm{H}^{c}$ \\
\hline $\mathrm{C}$ & 6.55 & -0.66 & -0.77 & -1.84 & -0.02 & $-0.05 \pm 0.15$ \\
$\mathrm{~N}$ & 5.60 & -1.61 & -1.56 & -2.18 & -0.36 & $-0.40 \pm 0.30$ \\
$\mathrm{O}$ & 7.21 & - & - & -1.45 & 0.37 & $0.52 \pm 0.15$ \\
$\mathrm{Ne}$ & 6.39 & -0.82 & -0.80 & -1.45 & 0.37 & - \\
$\mathrm{Si}$ & 5.89 & -1.32 & -1.46 & -1.62 & 0.20 & $0.21 \pm 0.08$ \\
$\mathrm{~S}$ & 5.57 & -1.64 & -1.55 & -1.57 & 0.25 & $0.21 \pm 0.07$ \\
$\mathrm{Ar}$ & 4.97 & -2.24 & -2.16 & -1.55 & 0.27 & - \\
$\mathrm{Fe}$ & 5.63 & -1.58 & -1.45 & -1.82 & - & - \\
\hline
\end{tabular}

${ }^{a} 12+\log (\mathrm{X} / \mathrm{H})$ by number in present model $M$.

${ }^{b}$ Empirical abundance: Izotov \& Thuan $(1998,1999)$ with $12+$ $\log (\mathrm{O} / \mathrm{H})=7.16$; Fe: Thuan \& Izotov (2005).

${ }^{c}$ Halo stars $[\mathrm{Fe} / \mathrm{H}] \sim-1.8$ : Fabbian et al. (2006); García Pérez et al. (2006); Nissen et al. (2007); Carbon et al. (1987).

predicted [Fe IV] 4906 and $\mathrm{Fe} / \mathrm{H}$ are divided by 1.4. This lower $\mathrm{Fe} / \mathrm{H}$ is adopted.

Solar abundances are tabulated by Asplund et al. (2005, AGS05). The compilation by Lodders (2003) is in agreement with AGS05 (+0.03 dex for all O-S elements of interest here and +0.02 dex for Fe relative to $\mathrm{H})$, except for $\mathrm{Ar} / \mathrm{H}(+0.37 \mathrm{dex})$. The larger argon abundance is convincingly advocated by Lodders (2003). Ar/O is adopted from this reference. Then $\mathrm{Ar} / \mathrm{H}$ coincides with the value listed by Anders \& Grevesse (1989). The shift of $\mathrm{O} / \mathrm{H}$ from Anders \& Grevesse (1989) to AGS05 is $-0.27 \mathrm{dex}$, out of which $-0.07 \mathrm{dex}$ corresponds to the change from proto-solar to solar abundances. Shifts for $\mathrm{X} / \mathrm{Fe}$ are $\sim-0.20$ dex for $\mathrm{N}, \mathrm{O}, \mathrm{Ne},-0.11 \mathrm{dex}$ for $\mathrm{C},-0.07$ dex for $\mathrm{S}$ and $\sim 0.0$ for other elements of interest.

In Table 7 , the present model abundances by number $12+\log (\mathrm{X} / \mathrm{H})$ for I Zw $18 \mathrm{NW}$ (“ $M$ ”) are provided in Col. 2. The abundances X/O relative to oxygen from models (Col. 3, " $M$ ") are compared to empirical values obtained by IT99 (Col. 4, "IT"). The brackets $[\mathrm{X} / \mathrm{Y}]=\log (\mathrm{X} / \mathrm{Y})-\log (\mathrm{X} / \mathrm{Y})_{\odot}$ from models are given in Cols. 5 and $6(\mathrm{Y} \equiv \mathrm{H}$ and $\mathrm{Fe})$. $[\mathrm{X} / \mathrm{Fe}]$ is provided for Galactic Halo stars with $[\mathrm{Fe} / \mathrm{H}] \sim-1.8$ (Col. 7, "H"). Despite considerable efforts to include $3 \mathrm{D}$ and non-LTE effects in the study of line formation in cool stars, astrophysical descriptions and atomic data may still entail uncertainties in stellar abundances (e.g., Fabbian et al. 2006), particularly for nitrogen. A population of N-rich stars is identified (e.g., Carbon et al. 1987).

Comparing Cols. 3 and 4, the model and empirical X/O agree to about 0.1 dex. The present $\mathrm{C} / \mathrm{O}$ is close to the one obtained by Garnett et al. (1997), who claim that C/O is anomalously large in IZw 18. IT99 argue that the subregion of I Zw $18 \mathrm{NW}$ observed with the HST is especially hot according to spatially resolved MMT data and that C/O is therefore small. Nonetheless, IT99 also derive an exceedingly low $\mathrm{O} / \mathrm{H}$ at the same position "because of the higher $T_{\mathrm{e}}$ ", which poses a problem of logic since there is a priori no link of causality between $T_{\mathrm{e}}$ and $\mathrm{O} / \mathrm{H}$ within $\mathrm{IZw} 18$. The $[\mathrm{C} / \mathrm{O}]=-0.39$ resulting from the present model is indeed marginally incompatible with the up-to-date $[\mathrm{C} / \mathrm{O}]=$ $-0.57 \pm 0.15$ corresponding to Galactic Halo stars with $[\mathrm{O} / \mathrm{H}]=$ -1.45 (Fabbian et al. 2006). This "large" [C/O] is analysed by Garnett et al. (1997) in terms of carbon excess, suggesting that an old stellar population managed to produce this element, then challenging the view that I Zw 18 is genuinely young (e.g., IT04), a view also challenged by Aloisi et al. (2007). From models, $[\mathrm{C} / \mathrm{Fe}]$ appears to be identical in $\mathrm{IZw} 18$ and halo stars of similar metallicity (Cols. 6 and 7). The relatively large $[\mathrm{C} / \mathrm{O}]$ in 
$\mathrm{IZw} 18$ is due to a relatively small [O/Fe]. This is indirectly confirmed by the agreement between I Zw 18 and halo stars for all elements beyond neon (argon should follow lighter $\alpha$-elements). The $[\mathrm{X} / \mathrm{O}] \mathrm{s}(\equiv[\mathrm{X} / \mathrm{H}]+1.45)$ are the usual basis to discuss elemental abundances in BCDs and nebulae. Exceptionally, in IZw 18, the abundances of iron and heavy $\alpha$-elements agree, allowing us to consider the oxygen abundance with respect to metallicity, instead of defining metallicity by means of oxygen itself. Any iron locked into dust grains would further decrease $[\mathrm{O} / \mathrm{Fe}]$ in IZw 18 . Apparently, for sufficiently low metallicity of the ISM and/or sufficient youth of the host galaxy, iron does not find paths to efficiently condense into dust. Alternatively, dust grains may be destroyed by shocks.

\subsection{Overall evaluation of models}

Line intensities are generally well accounted for, although [S IV] $\lambda 10.5 \mu$ is overpredicted by $90-100 \%$ (Sect. 6.4.3).

The freedom left in the parameters describing the SED and the shell acts at the few $\%$ level upon the [O III] $\lambda 4363$ predicted intensity. Thus, the calculated [O III] $\lambda 4363$ shifts from $96.0 \%$ of the observed intensity in $M 2\left(T 2=4 \times 10^{4} \mathrm{~K}, f_{i}^{\text {cov }}=0.26\right.$, $0.30)$ to $99.6 \%$ in $M 2 c\left(f_{i}^{\text {cov }}=0.22,0.60\right)$ and $99.8 \%$ in $\mathrm{M} 3$ $\left(T 2=5 \times 10^{4} \mathrm{~K}, f_{i}^{\mathrm{cov}}=0.23,0.50\right)$. Adding less than $1 \% \mathrm{lu}-$ minosity as soft X-rays (e.g., $M 2_{\mathrm{X}}$, compared to $M 2$, Sect. 6.5) results in $+1.7 \%$ for $[\mathrm{O} \mathrm{III}] \lambda 4363$. Also, increasing $\mathrm{He} / \mathrm{H}$ from 0.080 to the possibly more realistic value 0.084 (Peimbert et al. 2007), [O III] $\lambda 4363$ is enhanced by a further $+0.6 \%$. Since both the $\mathrm{X}$-ray and $\mathrm{He} / \mathrm{H}$ corrections are plausible, it is relatively easy to reach $100-102 \%$ of the observed [O III] $\lambda 4363$ intensity in the assumed configurations.

However, models tell us that $r([\mathrm{O} \mathrm{III}])$ can hardly be larger than the observed value. It may prove necessary to consider alternative gas distributions, e.g., if the [S IV] misfit is confirmed by more accurate observational and theoretical data. Assuming higher densities in the diffuse medium (Sect. 6.4.3) and/or considering thick filaments closer to the source tend to penalize [O III] $\lambda 4363$.

Uncertainties on [O III] collision strengths $\Omega$ need to be considered (Appendix D.2). Using $\Omega$ s by Lennon \& Burke (1994, LB94) instead of Aggarwal (1993, Ag93), the computed $\lambda 4363$ would be $2.1 \%$ smaller and more difficult to explain. On the other hand, using $\Omega\left({ }^{3} \mathrm{P}-{ }^{1} \mathrm{D}\right)$ from $\mathrm{Ag} 93$ and $\Omega\left({ }^{3} \mathrm{P}-{ }^{1} \mathrm{~S}\right)$ from LB94 would enhance all computed $\lambda 4363$ intensities by $2.1 \%$. Concerning transition ${ }^{3} \mathrm{P}-{ }^{1} \mathrm{~S}$ which controls $\lambda$ 4363, both Ag93 and LB94 find a $5-6 \%$ increase of $\Omega$ from $2 \times 10^{4} \mathrm{~K}$ to $3 \times$ $10^{4} \mathrm{~K}$, due to resonances. If for some reason the energy of these resonances would be shifted down, there would be room for a few $\%$ increase of $\Omega$ at $2 \times 10^{4} \mathrm{~K}$ compared to the current value, then introducing more flexibility in the present model of I Zw 18.

Thus, the hypothesis of pure photoionization by stars in the form explored here is strong, but the models approach a limit. This is satisfactory, considering that IZw 18 is an extreme object among BCDs, but sufficient flexibility in choosing solutions is worthwhile. An analysis of how the computed $r([\mathrm{O}$ III] $)$ can be influenced shows that, in the case of I Zw 18, possible variations of "astrophysical" origin are of the same order as the uncertainties affecting the $\Omega$ s. Since the set of computed $r$ ([O III]) tends to be reduced by $2-3 \%$ relative to observations, it is legitimate to question the $\Omega$ s. The recent re-evaluation of the distance to I Zw 18 by Aloisi et al. (2007) may offer an "astrophysical alternative": multiplying $D, R_{\mathrm{i}}$ and $R_{\mathrm{f}}$ by $2^{1 / 2}$ and the luminosty by 2 , the relative volume increase leads to smaller $P_{\text {in }}$ and $\tau_{\mathrm{c}}$, and, after reconvergence, $[\mathrm{O} \mathrm{III}] \lambda 4363$ is enhanced by $+2.4 \%$. Nonetheless, [S IV] $10.5 \mu$ is enhanced too $(+5 \%)$.

\section{Concluding remarks}

Due to its small heavy element content, IZw 18 stands at the high- $T_{\mathrm{e}}$ boundary of photoionized nebulae. Where ionization and temperature are sufficiently high, the cooling is little dependent on conditions, except through the relative concentration of $\mathrm{H}^{0}$, controlled by density. Therefore, from a photoionization model standpoint, $T_{\mathrm{e}}$ is then a density indicator, in the same way that it is an $\mathrm{O} / \mathrm{H}$ indicator in the usual $\mathrm{H} \mathrm{II}$ regions. It is for not having recognized the implications of this new logic that lowmetallicity BCD models failed.

In a photoionization model study of IZw $18 \mathrm{NW}, \mathrm{SS} 99 \mathrm{em}-$ ployed a filling-factor description and concluded that $T_{\mathrm{e}}$ ([O III]) was fundamentally unaccountable for. This description of the ionized gas owes its popularity to its simplicity and to its apparent success for usual H II regions. This success is no warranty for exactness, however, since it is based on the strong dependence of gas cooling on abundances and the filling-factor concept fails when applied to low-Z GEHIIRs. This conclusion of SS99 and other authors should be paralleled with the " $t$ problem" (Esteban et al. 2002; Peimbert et al. 2004), which calls into question the assumption of photoionization by stars as the overwhelmingly dominant source of heat in gaseous nebulae since the presence of $T_{\mathrm{e}}$ fluctuations supposedly larger than those reachable under this assumption implies additional heating.

A conclusion of the present study is that the gas distribution is no less critical than the radiation source in determining the line spectrum of H II regions. Assuming pure photoionization by stars, the implication of the large $T_{\mathrm{e}}([\mathrm{OIII}])$ of I Zw $18 \mathrm{NW}$ is that the mean density of the [O III] emitting region is much less than $N_{\mathrm{e}}$ ([S II]), a low $N_{\mathrm{e}}$ confirmed by line ratios [O IV] $\lambda 25.9 \mu / \mathrm{He} \mathrm{II} \lambda 4686$ and [Fe III] $\lambda$ 4986/[Fe III] $\lambda 4658$. I Zw $18 \mathrm{NW}$ models comprising a plausible SED and respecting geometrical constraints can closely match almost all observed lines from UV to IR, including the crucial [O III] $\lambda 4363$ ([S IV] $\lambda 10.5 \mu$ is a factor of 2 off, however). Thus, extra heating by, e.g., dissipation of mechanical energy in the photoionized gas of low-metallicity BCD galaxies like IZw 18 is not required to solve the " $T_{\mathrm{e}}([\mathrm{O} \mathrm{III}])$ problem". Moreover, since low-ionization fine-structure lines observed in I Zw 18 can be explained by soft $\mathrm{X}$-rays, (hydrodynamical) heating is not required either in warm $\mathrm{H}$ I regions protected from heating by star radiation.

The solutions found here are marginally consistent with observed $r([\mathrm{O} I I I])$. Given the claimed accuracy in the different fields of physics and astrophysics involved, postulating a mechanical source of heating is premature, whereas a 2-3\% upward correction to the collision strength for transition $\mathrm{O}^{2+}\left({ }^{3} \mathrm{P}-{ }^{1} \mathrm{~S}\right)$ at $T_{\mathrm{e}} \sim 2 \times 10^{4} \mathrm{~K}$ is an alternative worth exploring by atomic physics. Another possibility is a substantial increase of the distance to I Zw 18. From accurate spectroscopy and the peculiar conditions in IZw 18, astrophysical developments are at stake in the $5 \%$ uncertainty attached to [O III] collision strengths.

If photoionized nebulae are shaped by shocks and other hydrodynamic effects, this does not imply that the emission-line intensities are detectably influenced by the thermal energy deposited by these processes. Unravelling this extra thermal energy by means of spectroscopic diagnostics and models is an exciting prospect whose success depends on a recognition of all resources of the photoionization paradigm. Adopting the view 
that photoionization by radiation from young hot stars, including WR stars, is the only excitation source of nebular spectra in BCD galaxies, yet without undue simplifications, may help progress in the studies of stellar evolution, stellar atmosphere structure, stellar supercluster properties, giant H II region structure and finally possible sources of extra thermal energy.

\section{References}

Abrahamsson, E., Krems, R. V., \& Dalgarno, A. 2007, ApJ, 654, 1171 Aggarwal, K. M. 1983, ApJS, 52, 387

Aggarwal, K. M. 1993, ApJS, 85, 197 (Ag93)

Aggarwal, K. M., \& Keenan, F. P. 1999, ApJS, 123, 311

Aloisi, A., Clementini, G., Tosi, M., Annibali, F., et al. 2007, ApJ, 667, L151

Anders, E., \& Grevesse, N. 1989, Geochim. Cosmochim. Acta, 53, 197

Anderson, H., Ballance, C. P., Badnell, N. R., \& Summers, H. P. 2000, J. Phys. B, 33, 1255 (+ Erratum 2002, J. Phys. B, 35, 1613)

Asplund, M., Grevesse, N., \& Sauval, A. J. 2005, Cosmic Abundances as Records of Stellar Evolution and Nucleosynthesis, ed. T. G. Barnes, \& F. N. Bash, ASP Conf., 336, 25

Badnell, N. R. 1991, ApJ, 379, 356

Badnell, N. R. 2006, ApJS, 167, 334

Bakes, E. L. O., \& Tielens, A. G. G. M. 1994, ApJ, 427, 822

Barinovs, G., van Hemert, M. C., Krems, R., et al. 2005, ApJ, 620, 537

Barragán, P., Errea, L. F., Méndez, L., et al. 2006, ApJ, 636, 544

Brown, T. M., Heap, S. R., Hubeny, I., Lanz, T., \& Lindler, D. 2002, ApJ, 579L

Burke, V. M., Lennon, D. J., \& Seaton, M. J. 1989, MNRAS, 236, 353

Callaway, J. 1994, ADNDT, 57, 9

Campbell, A. 1990, ApJ, 362, 100

Cannon, J. M., Skillman, E. D., Garnett, D. R., \& Dufour, R. J. 2002, ApJ, 565, 931 (CSGD02)

Carbon, D. F., Barbuy, B., Kraft, R. P., et al. 1987, PASP, 99, 335

Cerviño, M., \& Luridiana, V. 2006, A\&A, 451, 475

Cerviño, M., Mas-Hesse, J. M., \& Kunth, D. 2002, A\&A, 392, 19

Cerviño, M., Luridiana, V., Pérez, E., et al. 2003, A\&A, 407, 177

Chambaud, G., Levy, B., Millie, P., et al. 1980, J. Phys. B, 13, 4205

Crowther, P. A. 2007, ARA\&A, 45, 177

Crowther, P. A., \& Hadfield, L. J. 2006, A\&A, 449, 711

Davidson, K., \& Kinman, T. D. 1985, ApJS, 58, 321

de Mello, D. F., Schaerer, D., Heldmann, J., \& Leitherer, C. 1998, ApJ, 507, 199

Dufour, R. J., \& Hester, J. J. 1990, ApJ, 350, 149

Dufour, R. J., Garnett, D. R., \& Shields, G. A. 1988, ApJ, 332, 752

Eissner, W., \& Seaton, M. J. 1974, J. Phys. B, 7, 2533

Eissner, W., Martins, P. de A. P., Nussbaumer, H., et al. 1969, MNRAS, 146, 63

Esteban, C., Peimbert, M., Torres-Peimbert, S., et al. 2002, ApJ, 581, 241

Fabbian, D., Asplund, M., Carlsson, M., \& Kiselman, D. 2006, A\&A, 458, 899

Galavis, M. E., Mendoza, C., \& Zeippen, C. J. 1995, A\&AS, 111, 347

Galavis, M. E., Mendoza, C., \& Zeippen, C. J. 1997, A\&AS, 123, 159 (GMZ97)

García Pérez, A. E., Asplund, M., Primas, F., et al. 2006, A\&A, 451, 621

García-Vargas, M. L., González-Delgado, R. M., Pérez, E., et al. 1997, ApJ, 478, 112

Garnett, D. R., Skillman, E. D., Dufour, R. J., et al. 1997, ApJ, 481, 174

Giammanco, C., Beckman, J. E., Zurita, A., \& Relaño, M. 2004, A\&A, 424, 877

González Delgado, R. M., \& Pérez, E. 2000, MNRAS, 317, 64

González Delgado, R. M., García-Vargas, M. L., Goldader, J., Leitherer, C., \& Pasquali, A. 1999, ApJ, 513, 707

Gräfener, G., \& Hamann, W.-R. 2005, A\&A, 432, 633

Guseva, N. G., Izotov, Y. I., \& Thuan, T. X. 2000, ApJ, 531, 776

Guseva, N. G., Izotov, Y. I., \& Thuan, T. X. 2006, ApJ, 644, 890

Henney, W. J., \& O’Dell, C. R. 1999, AJ, 118, 2350

Hirashita, H., \& Hunt, L. K. 2006, A\&A, 460, 67

Hunt, L. K., Dyer, K. K., \& Thuan, T. X. 2005, A\&A, 436, 837

Hunter, D. A., \& Thronson, H. A., Jr. 1995, ApJ, 452, 238

Izotov, Y. I., \& Thuan, T. X. 1998, ApJ, 497, 227

Izotov, Y. I., \& Thuan, T. X. 1999, ApJ, 511, 639 (IT99)

Izotov, Y. I., \& Thuan, T. X. 2004, ApJ, 616, 768 (IT04)

Izotov, Y. I., Foltz, C. B., Green, R. F., et al. 1997a, ApJ, 487, L37

Izotov, Y. I., Thuan, T. X., \& Lipovetsky, V. A. 1997b, ApJS, 108, 1

Izotov, Y. I., Chaffee, F. H., Foltz, C. B., et al. 1999, ApJ, 527, 757 (ICF99)

Izotov, Y. I., Chaffee, F. H., Foltz, C. B., et al. 2001a, ApJ, 560, 222

Izotov, Y. I., Chaffee, F. H., \& Schaerer, D. 2001b, A\&A, 378, L45

Izotov, Y. I., Stasińska, G., Meynet, G., et al. 2006a, A\&A, 448, 955

Izotov, Y. I., Schaerer, D., Blecha, A., et al. 2006b, A\&A, 459, 71
Jamet, L., Stasińska, G., Pérez, E., et al. 2005, A\&A, 444, 723 (JS05) Kaufman, M. J., Wolfire, M. G., \& Hollenbach, D. J. 2006, ApJ, 644, 283 Kunth, D., \& Östlin, G. 2000, A\&AR, 10, 1

Legrand, F., Kunth, D., Roy, J.-R., et al. 1997, A\&A, 326, L17

Leitherer, C. 2006, ASP Conf., Mass loss from stars and the evolution of stellar clusters, in press [arXiv: astro-ph/0608698]

Leitherer, C., Schaerer, D., Goldader, J. D., et al. 1999, ApJS, 123, 3

Lennon, D. J., \& Burke, V. M. 1994, A\&AS, 103, 273 (LB94)

Leroy, A., Cannon, J., Walter, F., et al. 2007, ApJ, 663, 990

Lodders, K. 2003, ApJ, 591, 1220

Luridiana, V., \& Peimbert, M. 2001, ApJ, 553, 633 (LP01)

Luridiana, V., Peimbert, M., \& Leitherer, C. 1999, ApJ, 527, 110 (LPL99)

Luridiana, V., Cerviño, M., \& Binette, L. 2001, A\&A, 379, 1017

Luridiana, V., Peimbert, A., Peimbert, M., et al. 2003, ApJ, 592, 846 (LPPC03)

Maciejewski, W., Mathis, J. S., \& Edgar, R. J. 1996, ApJ, 462, 347

Maeder, A., Meynet, G., \& Hirschi, R. 2005, The fate of the most massive stars, ed. R. M. Humphreys, \& K. Z. Stanek, ASP Conf., 332, 3

Martin, C. L. 1996, ApJ, 465, 680

Martin, C. L. 1997, ApJ, 491, 561

Mendoza, C. 1983, Planetary Nebulae, ed. D. Flower (Reidel), IAU Symp., 103, 143

Meynet, G., \& Maeder, A. 2005, A\&A, 429, 581

Nahar, S. N. 2000, ApJS, 126, 537

Nissen, P. E., Akerman, C., Asplund, M., et al. 2007, A\&A, 469, 319

Oey, M. S., Dopita, M. A., Shields, J. C., \& Smith, R. C. 2000, ApJS, 128, 511

Östlin, G. 2000, ApJ, 535, L99

Papaderos, P., Izotov, Y. I., Thuan, T. X., et al. 2002, A\&A, 393, 461

Peimbert, M. 1967, ApJ, 150, 825

Peimbert, M. 1995, in The analysis of emission lines, ed. R. Williams, \& M. Livio (Cambridge Univ. Press), 165

Peimbert, M., Peimbert, A., Ruiz, M. T., \& Esteban, C. 2004, ApJS, 150, 431

Peimbert, M., Luridiana, V., \& Peimbert, A. 2007, ApJ, 666, 636

Péquignot, D. 1990, A\&A, 231, 499

Péquignot, D., \& Tsamis, Y. G. 2005, A\&A, 430, 187

Péquignot, D., Ferland, G. J., Netzer, H., et al. 2001, Spectroscopic Challenges of Photoionized Plasmas, ed. G. J. Ferland, \& D. W. Savin, ASP Conf., 247, 533

Pradhan, A. K., Montenegro, M., Nahar, S. N., et al. 2006, MNRAS, 366, L6

Przybilla, N., \& Butler, K. 2004, ApJ, 610, L61

Relaño, M., Peimbert, M., \& Beckman, J. 2002, ApJ, 564, 704

Saraph, H. E., \& Storey, P. J. 1999, A\&AS, 134, 369

Saraph, H. E., Seaton, M. J., \& Shemming, J. 1969, Phil. Tr. R. Soc., A264, 77

Sargent, W. L. W., \& Searle, L. 1970, ApJ, 162, L155

Schlegel, D. J., Finkbeiner, D. P., \& Davis, M. 1998, ApJ, 500, 525

Seaton, M. J. 1958, Rev. Mod. Phys., 30, 979

Skillman, E. D., \& Kennicutt, R. C., Jr. 1993, ApJ, 411, 655 (SK93)

Stasińska, G., \& Izotov, Y. 2003, A\&A, 397, 71 (SI03)

Stasińska, G., \& Leitherer, C. 1996, ApJS, 107, 661

Stasińska, G., \& Schaerer, D. 1999, A\&A, 351, 72 (SS99)

Stasińska, G., Schaerer, D., \& Leitherer, C. 2001, A\&A, 370, 1

Stevens, I. R., \& Strickland, D. K. 1998, MNRAS, 301, 215

Stevenson, C. C., McCall, M. L., \& Welch, D. L. 1993, ApJ, 408, 460

Strickland, D. K., \& Stevens, I. R. 1999, MNRAS, 306, 43

Tayal, S. S. 2000, ApJ, 530, 1091

Tayal, S. S. 2006, ApJS, 166, 634

Tayal, S. S. 2006b, J. Phys. B, 39, 4393

Tayal, S. S., \& Gupta, G. P. 1999, ApJ, 526, 544

Tenorio-Tagle, G. 1996, AJ, 111, 1641

Tenorio-Tagle, G., Muñoz-Tuñón, C., Pérez, E., et al. 2006, ApJ, 643, 186

Thompson, R. I., Sauvage, M., Kennicutt, R. C., Jr., et al. 2006, ApJ, 638, 176

Thuan, T. X., \& Izotov, Y. I. 2005, ApJS, 161, 240 (TI05)

Thuan, T. X., Bauer, F. E., Papaderos, P., \& Izotov, Y. I. 2004, ApJ, 606, 213

Tsamis, Y. G., \& Péquignot, D. 2005, MNRAS, 364, 687

van Zee, L., Westpfahl, D., Haynes, M. P., \& Salzer, J. J. 1998, ApJ, 115, 1000

Veilleux, S., Cecil, G., \& Bland-Hawthorn, J. 2005, ARA\&A, 43, 769

Vidal-Madjar, A., Kunth, D., Lecavelier des Etangs, A., et al. 2000, ApJ, 538, 77

Viegas, S. M. 2002, RMxA\&A (Ser. de Conf.), 12, 219 (V02)

Vílchez, J. M., \& Iglesias-Páramo, J. 1998, ApJ, 508, 248 (VI98)

Vink, J. S., \& de Koter, A. 2005, A\&A, 442, 587

Weise, W. L., Fuhr, J. R., \& Deters, T. M. 1996, J. Phys. Chem. Ref. Data, Monograph, 7

Wöste, G., Wunner, G., Noble, C. J., et al. 2002, J. Phys. B, 35, 4847

Wu, Y., Charmandaris, V., Hao, L., et al. 2006, ApJ, 639, 157

Wu, Y., Charmandaris, V., Hunt, L. K., et al. 2007, ApJ, 662, 952 (Wu07) 
D. Péquignot: Heating of IZw 18, Online Material p 1

\section{Online Material}




\section{Appendix A: Models for other GEHIIRs}

Models of GEHIIRs include studies of individual objects and evolutionary sequences for large samples. Examples ordered by decreasing $\mathrm{O} / \mathrm{H}$ are reviewed.

\section{A.1. Individual GEHIIRs}

\section{A.1.1. $\mathrm{O} / \mathrm{H} \geq 1.5 \times 10^{-4}$}

González Delgado \& Pérez (2000) successfully model NGC 604 $\left(\mathrm{O} / \mathrm{H}=3 \times 10^{-4}\right)$ in M 33 as a radiation-bounded sphere (radius $20-110 \mathrm{pc}$ ) of density $30 \mathrm{~cm}^{-3}$ and filling $\epsilon=0.1$, both [O I $] \lambda 6300$ and [O III] $\lambda 4363$ being explained.

García-Vargas et al. (1997) successfully model circumnuclear GEHIIRs in NGC 7714 as thin, constant-density $\left(N_{\mathrm{H}} \sim 200 \mathrm{~cm}^{-3}\right)$, radiation-bounded shells with $\mathrm{O} / \mathrm{H}=(2-3) \times$ $10^{-4}: r([\mathrm{O} \mathrm{III}])$ is accounted for within errors and $[\mathrm{OI}]$ is just moderately underestimated. The nuclear GEHIIR of NGC 7714 is modelled by González Delgado et al. (1999) as a full sphere with very small $\epsilon$. The adopted $\mathrm{O} / \mathrm{H}=3 \times 10^{-4}$ is too large since $[\mathrm{O}$ III $] \lambda 4363$ is underpredicted. Obviously, a better fit to the available optical line spectrum could be achieved for the nucleus. González Delgado et al. (1999) are probably not well founded to invoke extra heating by shocks.

Luridiana \& Peimbert (2001, LP01) propose a photoionization model for NGC $5461\left(\mathrm{O} / \mathrm{H}=2.5 \times 10^{-4}\right)$, a GEHIIR in M 101. As for NGC 2363 (Appendix A.1.2), LP01 apply an aperture correction to their spherical model. The $\mathrm{H} \beta$ and $r$ ([S II]) spatial profiles are reproduced with a Gaussian density distribution of very small $\epsilon$ and high inner density $-500 \mathrm{~cm}^{-3}$ compared to $N_{\mathrm{e}}([\mathrm{S} \mathrm{II}]) \sim 150 \mathrm{~cm}^{-3}-$ meant to increase the inner $\mathrm{O}^{+}$fraction. In this way, the $\lambda 5007, \lambda 4363$ and $\lambda 3727$ fluxes restricted to the theoretical slit can be accounted for ${ }^{5}$, but not [O I], "a not unusual fact", nor [S II], which, against a statement of LP01, is not enhanced by increasing the primary flux below 1.0 ryd. As noted by LP01, the outputs of their model are strongly dependent on the density structure.

From their sophisticated study of NGC $588(\mathrm{O} / \mathrm{H}=2 \times$ $10^{-4}$ ), Jamet et al. (2005, JS05) conclude that "the energy balance remains unexplained". This negative conclusion is based on the fact that $T_{\mathrm{e}}([\mathrm{O}$ III $])$, from the ratio $\lambda 4363 \AA / \lambda 5007 \AA$, is observed to be larger than $T_{\mathrm{e}}([\mathrm{O}$ III] $]$ IR), from $\lambda 5007 \AA / \lambda 88 \mu \mathrm{m}$, by $\Delta T_{\text {obs }}=2700 \pm 700 \mathrm{~K}$, while the corresponding $\Delta T_{\text {mod }}$ is only $1400 \pm 200 \mathrm{~K}$ in models which are otherwise satisfactory, accounting reasonably well for $T_{\mathrm{e}}([\mathrm{O} \mathrm{III}])$ and the distribution of ionization (models DD1 and DDH exhibited by JS05, who carefully consider uncertainties related to the SED and the smallscale gas distribution). Considering the difficulty of calibrating the ISO-LWS fluxes relative to the optical and the sensitivity of the [O III] $\lambda 88 \mu \mathrm{m}$ emissivity to $N_{\mathrm{e}}$, the $400 \mathrm{~K}$ gap between $\Delta T_{\mathrm{obs}}$ and $\Delta T_{\text {mod }}$ is not a sound basis to claim the existence of an energy problem. If diagnostics based on IR lines are desirable, the energy problem raised so far in GEHIIR studies is not related to these lines. Instead of a heating problem as in I Zw 18, the model presented by JS05 could be facing a cooling problem, since the computed $T_{\mathrm{e}}$ ([O III], IR) is too high.

An additional energy source is not needed in the cases of NGC 588, NGC 5461, NGC 7714 and NGC 604.

5 LP01 state a priori that [O III] $\lambda 4363$ "almost surely has a contribution from processes other than photoionization" and conclude that their model "fails to reproduce the observed [O III] $\lambda 4363$ intensity", but both statements seem to be refuted by evidence they present.

\section{A.1.2. $\mathrm{O} / \mathrm{H}<1.5 \times 10^{-4}$}

Relaño et al. (2002) provide an inventory of NGC 346, a GEHIIR of the SMC $\left(\mathrm{O} / \mathrm{H}=1.3 \times 10^{-4}\right)$. Their spherical, constantdensity, matter-bounded photoionization model, whose only free parameter is a filling factor $\epsilon$ (alla SS99), accounts for the escape of ionizing photons, but underpredicts collisional lines, especially $[\mathrm{O} \mathrm{III}] \lambda 4363$. After unsuccessful variations on geometry, the authors preconize, following SS99, an additional source of energy.

After an extensive exploration of photoionization models with filling factor for the bright GEHIIR NGC $2363(\mathrm{O} / \mathrm{H}=$ $8 \times 10^{-5}$ ), Luridiana et al. (1999, LPL99) conclude that they cannot find a solution unless they introduce $T_{\mathrm{e}}$ fluctuations by hand, i.e., they assume a larger $t^{2}$ than the one intrinsic to their model. This $t^{2}$, intended to enhance the computed [O III] $\lambda 4363$, is justified by the fact that the observed Paschen jump temperature is less than $T_{\mathrm{e}}([\mathrm{O} \mathrm{III}])$ and supported by a self-consistency argument: a larger $t^{2}$ leads to a larger $\mathrm{O} / \mathrm{H}$, hence a larger number of WR stars, hence (1) a larger injection of mechanical energy, supposed to feed the temperature fluctuations themselves, and (2) a higher photon flux above the $\mathrm{He}^{+}$ionization limit, useful to increase He II $\lambda$ 4686. However, as acknowledged by Luridiana et al. (2001), the WR star winds generate an insufficiently large $t^{2}$ in NGC 2363. Also, present views suggest that arguments based on WR stars in low-Z galaxies were false (e.g., Leitherer 2006; Appendix C). Finally, $r$ ([O III]), underestimated by only $\sim 12 \%$ in the "standard" low-Z model by LPL99, is divided by 2 on using the larger $\mathrm{O} / \mathrm{H}$, so that a relatively minor difficulty is made much worse and then solved by means of an arbitrary $t^{2}$. The slit correction advocated by LPL99 is considered in Appendix B.2.

Luridiana et al. (2003, LPPC03) consider a spherical model for a GEHIIR of SBS 0335-052 $\left(\mathrm{O} / \mathrm{H}=2 \times 10^{-5}\right)$. A Gaussian distribution with high maximum density and small $\epsilon$ proves unsatisfactory. LPPC03 then consider a 10-shell model (over 50 free parameters, most of which are pre-defined), in which each shell is radiation bounded and is characterized by a covering factor. Although each shell is still given an $\epsilon$, the new model is equivalent to a collection of geometrically thin radiationbounded sectors at different distances from the source (see also Giammanco et al. 2004) and "gracefully reproduces the constancy of the ionization degree along the diameter of the nebula". Hence, the authors are forced by observational evidence to implicitly abandon the classical filling-factor approach. Nonetheless, whatever the complexity of these models, all of them fail to account for the high $T_{\mathrm{e}}$ ([O III]).

LPPC03 consider a Gaussian model for IZw $18 \mathrm{SE}(\mathrm{O} / \mathrm{H}=$ $1.7 \times 10^{-5}$ ) with again a relatively large maximum density and, unlike for SBS 0335-052, a relatively large $\epsilon$, resulting in a rather compact model nebula, in which the computed $T_{\mathrm{e}}$ ([O III]) compares quite well with the observed one. Unlike for the NW, the HST image (Cannon et al. 2002) of the younger SE H II region does not show a shell surrounding an MSC. Nonetheless, considering the strong output of mechanical energy from massive stars, it is likely that inner cavities already developed. The strong indirect evidence for too compact a gas distribution in the model by LPPC03 is the notable weakness of the computed intensity of [O II] and other low-ionization lines. Adopting a more expanded structure in order to increase [O II], yet keeping the general trend of the gas distribution, the computed $T_{\mathrm{e}}([\mathrm{O} \mathrm{III}])$ would be forseeably lower than in the model by LPPC03.

Previous photoionization models for low oxygen abundance GEHIIRs appear to systematically fail. 


\section{A.2. Individual GEHIIRs: discussion}

LPPC03 describe the " $T_{\mathrm{e}}$ ([O III]) problem" they face in their study of SBS 0335-052 (Appendix A.1.2) as "a systematic feature" of H II region models and, following SS99, they state that this problem "can be ascribed to an additional energy source acting in photoionization regions, other than photoionization itself". Nevertheless, $T_{\mathrm{e}}$ ([O III]) seems to be accountable in existing photoionization models for GEHIIRs with, say, $\mathrm{O} / \mathrm{H} \geq 1.5 \times$ $10^{-4}$ (Appendix A.1.1). Similarly, the computed [O III] $\lambda 4363$ is correct, possibly even too large, for H II regions of the LMC (Oey et al. 2000). If, despite apparent complementarities, the $T_{\mathrm{e}}([\mathrm{O} \mathrm{III}])$ and $t^{2}$ problems have different origins (Sect. 1), no "systematic feature" can be invoked.

In modelling near solar abundance GEHIIRs, [O III] $\lambda 4363$ is controlled by $\mathrm{O} / \mathrm{H},[\mathrm{O} \mathrm{III}] \lambda 5007$ by the "color temperature" of the ionizing radiation, [O II] $\lambda 3727$ by the ionization parameter, while $[\mathrm{OI}]$ is maximized in radiation-bounded conditions. For these objects, assuming a "large" (constant) density, e.g., $\geq N_{\mathrm{e}}$ ([S II]), associated with an ad hoc $\epsilon \ll 1$, is often successful, although this does not prejudge the relevance of the model found. Indeed, this assumption proves to be at the heart of the $T_{\mathrm{e}}([\mathrm{O} \mathrm{III}])$ problem met in low-Z BCDs (Appendix B.1).

\section{A.3. Extensive analyses of $B C D$ s}

The conclusion of an early extensive analysis based on radiation-bounded, low-density full sphere models for low-Z BCDs (Stasińska \& Leitherer 1996) is optimistic concerning $T_{\mathrm{e}}$ ([O III]), whereas [O I] is then qualitatively explained in terms of shock heating. Nevertheless, in an extension of this study to large-Z objects with no measured $T_{\mathrm{e}}([\mathrm{O} \mathrm{III}])$, Stasińska et al. (2001) reinforce the energy problem raised by SS99 (Sect. 2.2) when they conclude that "a purely "stellar" solution seems now clearly excluded for the problem of $[\mathrm{O} \mathrm{III}] / \mathrm{H} \beta$ versus $[\mathrm{O}$ II $] / \mathrm{H} \beta$ as well as $[\mathrm{S} \mathrm{II}] / \mathrm{H} \beta$ ", while, conversely, they still endorse the unproved statement of SS99 (Sect. 2.3) that "strong [O I] emission is easily produced by photoionization models in dense filaments".

The sequence of photoionization models proposed by Stasińska \& Izotov (2003, SI03) for a large sample of low-Z BCDs (divided in three abundance bins) illustrates views expressed after the failure of models acknowledged by SS99 for IZw 18 (Sect. 2). In the description by SI03, an evolving synthetic stellar cluster $\left(10^{5} M_{\odot}\right.$, instantaneous burst $)$ photoionizes a spherical shell of constant density $N_{\mathrm{H}}=10^{2} \mathrm{~cm}^{-3}$ at the boundary of an adiabatically expanding hot bubble. With suitable bubble properties, underlying old stellar population, aperture correction and time evolution of the covering factor, the range of $\mathrm{H} \beta$ equivalent width $(\mathrm{EW}(\mathrm{H} \beta))$ and the trends of [O III] 5007, [O II] 3727, [O I] 6300 versus $\mathrm{EW}(\mathrm{H} \beta)$ can be reproduced for the high- $\mathrm{Z}$ bin $\left(\mathrm{O} / \mathrm{H} \sim 1.5 \times 10^{-4}\right)$ within the scatter of the data.

Applying similar prescriptions to the intermediate- $Z$ bin, the oxygen lines and He II $\lambda 4686$ (He II was just fair in the first bin) are underpredicted. SI03 diagnose an insufficient average energy per absorbed photon and assume that the stellar cluster is supplemented by a strong $10^{6} \mathrm{~K}$ bremsstrahlung-like radiation source, which solves the $\mathrm{He}$ II problem $\left(\mathrm{He}^{+}\right.$is further ionized by extra 4-5 ryd photons; see, however, Appendix C) and alleviates the $[\mathrm{O} I]$ problem (the soft X-rays further heat and widen the ionization front), but barely improves [O III] and [O II]. Agreement of the model sequence with observation is finally restored by supposing in addition that the shell includes a time-variable oxygenrich gas component attributed to self-enrichment: in the example shown by SI03, this component is 4-fold enriched in CNO, etc. relative to the original abundance and encompasses half of the shell mass after a few Myrs, so that one generation of stars produced a 2.5 -fold enhancement of the average abundance in the photoionized gas.

This description essentially applies to the low metallicity bin $\left(\mathrm{O} / \mathrm{H} \sim 2 \times 10^{-5}\right)$ of particular concern for I Zw 18, but with even more extreme properties for the O-rich component, since it should be overabundant by $1 \mathrm{dex}$, resulting in a 5-fold enhancement of the final average abundance.

\section{A.4. Extensive analyses of BCDs: discussion}

The time scale of 0.5 Myrs for the growth of the O-rich component in the description by SI03 cannot directly fit in the selfpollution scenario since it is shorter than the stellar evolution time scale. Also, a sudden oxygen self-pollution of the gas is not observed in supernova remnants.

The assumed X-ray power is $\sim 10 \%$ of the cluster luminosity or $\sim 2$ dex times the estimated X-ray ROSAT power $(0.07-2.4 \mathrm{keV})$ of the hot bubble fed by stellar winds and supernovae around a usual MSC (Strickland \& Stevens 1999; Cerviño et al. 2002). Moreover, the hot gas is generally raised at several $10^{6} \mathrm{~K}$ (Stevens \& Strickland 1998). Adopting a larger temperature, the X-ray power should be even larger, as only the softer radiation interacts usefully with the ionized gas. IZw 18 itself is a rather strong $\mathrm{X}$-ray emitter in the $0.5-10 \mathrm{keV}$ range, yet 20 times weaker than the source assumed by SI03 (Thuan et al. 2004; Sect. 6.5).

Apart from these problems, SI03 do not address the question of the intensity of [O III] $\lambda$ 4363. The narrow radiation-bounded shell adopted by SI03 usefully favours [O I] $\lambda$ 6300, but makes the computed intensity of [O III] $\lambda 4363$ even worse than the one obtained by, e.g., SS99 (Sect. 2). Moreover, adding the prominent O-rich component advocated by SI03 will (1) decrease [O III] $\lambda 4363$ by a further $30-40 \%$ on average and (2) conflict with the existence of very low-Z BCDs, since any of them will shift to the intermediate class defined by SI03 after 1-2 Myrs.

If what SI03 qualify as "appealing explanations" are necessary for BCD models, then the hypothesis of photoionization by stars, which was already given a rough handling by SS99 in their analysis of IZw 18, should be considered as definitively excluded for the whole class of low-Z BCDs. The fact that SI03 discard [O III $] \lambda 4363$ in their analysis confirms that they endorse and reinforce views expressed by SS99 or Stasińska et al. (2001) and give up explaining $T_{\mathrm{e}}$ ([O III] $)$ in low-metallicity GEHIIRs by means of stellar radiation. However, the same restrictive assumption as for individual GEHIIR studies (Appendices A.1-A.2) bears on the gas distribution adopted by SI03, since their (geometrically thin) "high" constant- $N_{\mathrm{H}}$ model sphere is a zero-order approximation of a model shell with a classical filling factor (Appendix B.1).

\section{Appendix B: The gas distribution in GEHIIRs}

\section{B.1. The filling factor concept}

To reproduce the $\mathrm{H} \alpha$ surface brightness of $\mathrm{H}$ II regions, asssuming a gas density much larger than $\left\langle N_{\mathrm{e}}^{2}\right\rangle^{1 / 2}$, the "filling factor paradigm" posits that the emitting gas belongs to optically thin, "infinitesimal" clumps, filling a fraction $\epsilon \ll 1$ of the volume.

Given that the stellar evolution timescale exceeds the sound crossing time of $\mathrm{H}$ II regions, small optically thin ionized clumps will have time to expand and merge into finite-size structures. If 
these structures are assumed to have the original density, they are likely to have finite or large optical depths, in contradiction with the filling factor concept.

Filamentary structures, ubiquitous in $\mathrm{H} \alpha$ images of nearby GEHIIRs, are often taken as justification for introducing $\epsilon$ in photoionization models. However, (1) the geometrical thickness of observed filaments is consistent with radiation-bounded structures and (2) an individual filament most often emits both high and low ionization lines (e.g., Tsamis \& Péquignot 2005). The filling-factor description is flawed.

A GEHIIR may well be a collection of radiation-bounded filaments embedded in coronal and photoionized diffuse media. The idea behind assuming this configuration is that only the ionized "atmospheres" of long-lived, radiation-bounded, evaporating structures will maintain a substantial thermal overpressure relative to their surroundings (a similar idea applies to the "proplyds" found in Orion; e.g., Henney \& O'Dell 1999).

The filling factor concept fails on both theoretical and observational grounds. Nevertheless, introduced as a technical tool to manage $N_{\mathrm{e}}$ diagnostics like $r$ ([S II]), $\epsilon$ came to be improperly used to adjust the local ionization equilibrium of the gas through $N_{\mathrm{H}}$, in an effort to overcome problems of ion stratification generated by the filling factor description itself (Appendix B.2).

The $T_{\mathrm{e}}([\mathrm{O} \mathrm{III}])$ problem met in oxygen-poor GEHIIRs may relate to the loss of plasticity affecting photoionization models, as the dependence of gas cooling on abundances vanishes. Then, cooling depends on the relative concentration of $\mathrm{H}^{0}$ (collisional excitation of $\operatorname{Ly} \alpha$ ), controlled by the local $N_{\mathrm{H}}$. Hence, the (improper) freedom on $\epsilon$ is eroded. Moreover, if the density is not uniform, $N_{\mathrm{e}}([\mathrm{S} \mathrm{II}])$ is a biased estimate for $N_{\mathrm{e}}$ in the bulk of the emitting gas, since $\mathrm{S}^{+}$ions will belong to dense, optically thicker clumps. Emission from an interclump medium with $N_{\mathrm{e}}<$ $N_{\mathrm{e}}([\mathrm{S}$ II] $)$ will selectively enhance the computed [O III] $\lambda 4363$ intensity. LPL99 state that their model includes "denser condensations uniformly distributed in a more tenuous gas", but in practice only the condensations emit. This restriction is shared by virtually all published models for low-Z GEHIIRs. While the assumed density of the emitting gas can be orders of magnitude larger than $\left\langle N_{\mathrm{e}}^{2}\right\rangle^{1 / 2}$, emission from a lower density gas is neglected by construction (The study by JS05 is an exception, but NGC 588 is not low-Z; Appendix A.1.1). The $T_{\mathrm{e}}$ ([O III]) problem suggests lifting this restriction.

\section{B.2. Spheres, slits, filling factor and stratification}

Spherical models raise the question of how to compare computed spectra with nebular spectra observed through, e.g., a narrow slit. LPL 99 (Appendix A.1.2) advocate extracting emission from that part of the sphere which would project on the slit. Despite obvious problems with non-sphericity, LPL 99 and others argue that this procedure would at least allow weighting the contributions from low- and high-ionization zones in a more realistic manner. Using the classical filling factor concept (Appendix B.1) in GEHIIR models, ion stratification spreads over the whole nebula and the [OI] emission is effectively confined to outer layers, in which the primary radiation eventually vanishes. If, on the contrary, the emitting gas belongs to radiation-bounded filaments distributed within the nebula, then ion stratification disappears to first order. Radial ionization gradients, if any, are no longer related to a progressive destruction of primary photons along the full radial extension of the nebula, but to changes in (local) average ionization parameter.

LPL 99 conclude that [OI] is due to shock excitation in NGC 2363 because the computed intensity is weak in their theoretical slit extraction. Nonetheless, the [O I] intensity is fairly correct in their global spectrum. This apparent failure of their photoionization model may be due to the unfortunate combination of (1) a very small $\epsilon$ and (2) the extraction of a slit shorter than the diameter of the model sphere. Along the same lines, LPPC03 are confronted with undesirable consequences of the filling factor assumption on the variation of ionization along a slit crossing SBS 0335-052 (Appendix A.1.2).

If a geometrically defined model can hardly provide an approximation to a complex H II region, thus casting doubt on theoretical slit extractions, global spectra are less sensitive to geometry, because of conservation laws.

Moreover, in computing 1D photoionization models, the (spherical) symmetry enters only in the treatment of the diffuse ionizing radiation field, which is generally not dominant in the total field. The diffuse field, most effective just above the ionization limits of $\mathrm{H}, \mathrm{He}$ and $\mathrm{He}^{+}$, is relatively local at these photon energies (in accordance with the "Case B" approximation) and little dependent on global geometry. Let us define an "elementary spherical model" (for given SED) as a radial density distribution of whatever complexity. Since the local state of the gas is chiefly related to the primary (radial) radiation, a composite model made of a judicious combination of elementary spherical models, each of them restricted to a sector characterized by a covering factor, can provide topologically significant and numerically accurate descriptions of global spectra for nebulae with complex structures. Defining a "topology" as a particular set of spherical models with their attached covering factors, any given topology is in one-to-one correspondence with a global spectrum and a full class of geometries, since any sector can be replaced by an arbitrary set of subsectors, provided that the sum of the covering factors of these subsectors is conserved.

Thus, a good modelling strategy for a GEHIIR is one in which a global (probably composite) model spectrum is compared to the observed global spectrum. If only one slit observation is available, given that the ion stratification tends to be relatively loose and erratic in GEHIIRs, it is wise to directly use this spectrum as the average spectrum (together with scaling by the absolute $\mathrm{H} \beta$ flux), with the understanding that the resulting photoionization model will represent a "weighted average" of the real object. For many practical purposes, this weighting may not significantly impact on the inferences made from the model, unless the slit position is largely unrepresentative.

\section{Appendix C: Hell $\lambda$ 4686, WR stars and SEDs}

IZw 18 harbours Wolf-Rayet (WR) stars (Legrand et al. 1997; Izotov et al. 1997a; de Mello et al. 1998; Brown et al. 2002). WR stars have been challenged as the sole/main cause of nebular He II $\lambda 4686$ in BCDs on the basis of a lack of correlation between the occurence of this line and the broad "WR bumps" (e.g., Guseva et al. 2000). The study of WR stars is experiencing a revolution (Maeder et al. 2005; Meynet \& Maeder 2005; Gräfener \& Hamann 2005; Vink \& de Koter 2005; Crowther 2007) after the realization that (1) rotation of massive stars favours enhanced equatorial mass loss, element mixing by shears, and angular momentum transport by meridian circulation, (2) low-Z massive stars tend to be fast rotators and accelerate as they evolve off the main sequence, so that the lower mass limit for a star to become a WNE star is much reduced, and (3) for a given type of WR star, the mass loss is lower for lower metallicity $(\mathrm{Fe} / \mathrm{H}$, not $\mathrm{O} / \mathrm{H})$, with three consequences: the broad WR features are less evident for low metallicity (weaker optical continuum and smaller EW of WR bumps), the duration of the 
WR stage can be longer, and the EUV luminosity is larger due to a reduced blanketing effect. Thus, the above lack of correlation can now be partly ascribed to a bias, related to the tendency of WR star atmospheres to display less prominent optical signatures when they emit more EUV radiation. The WR star population of IZw 18 and the ability of these stars to emit radiation beyond 4 ryd have almost certainly been grossly underestimated (Crowther \& Hadfield 2006).

Other observations, e.g., for SBS 0335 052E (Izotov et al. 2001b, 2006b) are still taken as evidence for He II excitation by radiation from very fast shocks: (1) the He II line is broader than other nebular lines; (2) the He II emission is spread out far away from the main MSCs; and (3) $T_{\mathrm{e}}$ is larger in the He II emitting area, hence at large distances from the main ionizing sources. These findings are not compelling arguments against photoionization by WR stars. The larger He II line width indicates greater turbulence and/or velocity gradients, not necessarily shocks. That $T_{\mathrm{e}}$ is observed to be larger in He II emitting gas is in agreement with photoionization models. The spatial extent of He II may reflect the distribution of a few WR stars, which may not belong to the main cluster and may not be easily detected (Crowther \& Hadfield 2006). Alternatively, He II can be produced far from the ionizing stars if the medium is porous and permeated by low density, optically thin gas, e.g., along a galactic wind outflow (Izotov et al. 2006b). The picture of a galactic wind also suggests an explanation for the He II width.

Photoionization models are test beds for ionizing radiation sources, but inferences about the physics of GEHIIRs should not depend on uncertain SEDs. Existing synthetic star clusters are inadequate to model IZw 18. Apart from known problems with star sampling (Cerviño et al. 2003; Cerviño \& Luridiana 2006), limited knowledge of the history of actual MSCs and current uncertainties about WR stars, new free parameters (initial angular momentum and magnetic field of individual stars; rate of binarity) will broaden the range of possible SED evolutions, while collective effects in a compact cluster of massive stars may influence the output of ionizing radiation far from it, due to highdensity stellar winds (Thompson et al. 2004).

These comments justify (1) the assumption of an excitation of He II solely by WR stars and (2) the use of a flexible analytical SED for I Zw $18 \mathrm{NW}$ (Sect. 4.1).

\section{Appendix D: Atomic data}

\section{D.1. Collisional excitation of $\mathrm{HI}$}

Collision strengths $\Omega(1 s-n l)(n<6 ; l<n)$ for HI are taken from Anderson et al. (2000, ABBS00). The $\Omega$ s for $1 s-2 s$ and $1 s-2 p$ are much larger than for the next transitions $1 s-n l$ and are not controversial. The main cooling agent in low-Z BCDs should be correctly implemented in all codes. Nonetheless, in the conditions of IZw 18 , the results for transitions $1-2$ by ABBS00 are about $10 \%$ larger than those carefully fitted by Callaway (1994), giving an estimate of possible uncertainties. The adopted data tend to enhance the cooling with respect to earlier data and to (conservatively) worsen the " $T_{\mathrm{e}}$ ([O III]) problem". Total $\Omega(1-n)$ listed by Przybilla \& Butler (2004) virtually coincide with ABBS00 values for 1-2, confirming the $\mathrm{HI}$ cooling rate, but diverge from $\mathrm{ABBS} 00$ for $n>2$ and increasing $T_{\mathrm{e}}$ similarly to early, probably wrong, data (see Péquignot $\&$ Tsamis 2005).
Table D.1. Effective collision strengths for [O III].

\begin{tabular}{|c|c|c|c|c|}
\hline$T_{\mathrm{e}} / 10^{4} \mathrm{~K}:$ & 0.5 & 1.0 & 2.0 & 3.0 \\
\hline Reference: $^{a}$ & \multicolumn{4}{|c|}{$\Omega\left({ }^{3} \mathrm{P}-{ }^{1} \mathrm{D}\right)$} \\
\hline Sea58 & - & 1.59 & - & - \\
\hline SSS69 & - & 2.39 & - & - \\
\hline ENS69 & 1.85 & 2.50 & 2.91 & 2.96 \\
\hline ES74 & 2.17 & 2.36 & 2.55 & - \\
\hline Men83 & 2.02 & 2.17 & 2.39 & - \\
\hline Ag83 & 2.035 & 2.184 & 2.404 & 2.511 \\
\hline BLS89 & 2.10 & 2.29 & 2.51 & 2.60 \\
\hline Ag93 & 2.039 & 2.191 & 2.414 & 2.519 \\
\hline LB94 & 2.1268 & 2.2892 & 2.5174 & 2.6190 \\
\hline $\mathrm{AgK} 99^{b}$ & 2.0385 & 2.1906 & 2.4147 & 2.5191 \\
\hline $\mathrm{LB}^{\circ} / \mathrm{AgK} 99^{c}$ & \multicolumn{4}{|c|}{$\Omega\left({ }^{3} \mathrm{P}-{ }^{1} \mathrm{~S}\right)$} \\
\hline Sea58 & - & 0.220 & - & - \\
\hline SSS69 & - & 0.335 & - & - \\
\hline ENS69 & 0.255 & 0.298 & 0.331 & 0.339 \\
\hline ES74 & 0.276 & 0.325 & 0.356 & - \\
\hline Men83 & 0.248 & 0.276 & 0.314 & - \\
\hline Ag83 & 0.2521 & 0.2793 & 0.3162 & 0.3315 \\
\hline BLS89 & 0.260 & 0.287 & 0.318 & 0.331 \\
\hline Ag93 & 0.2732 & 0.2885 & 0.3221 & 0.3404 \\
\hline LB94 & 0.2720 & 0.2925 & 0.3290 & 0.3466 \\
\hline $\operatorname{AgK} 99^{b}$ & 0.2732 & 0.2885 & 0.3221 & 0.3404 \\
\hline $\mathrm{LB}^{\circ} / \mathrm{AgK} 99^{c}$ & \multicolumn{4}{|c|}{$\Omega\left({ }^{1} \mathrm{D}-{ }^{1} \mathrm{~S}\right)$} \\
\hline Sea58 & - & 0.640 & - & - \\
\hline SSS69 & - & 0.310 & - & - \\
\hline ENS69 & 0.483 & 0.578 & 0.555 & 0.510 \\
\hline ES74 & 0.807 & 0.856 & 0.752 & - \\
\hline Men83 & 0.516 & 0.617 & 0.634 & - \\
\hline Ag83 & 0.5463 & 0.6468 & 0.6670 & 0.6524 \\
\hline BLS89 & 0.59 & 0.677 & 0.664 & 0.634 \\
\hline Ag93 & 0.4312 & 0.5227 & 0.5769 & 0.5812 \\
\hline LB94 & 0.4942 & 0.5815 & 0.6105 & 0.6044 \\
\hline $\operatorname{AgK} 99^{b}$ & 0.4312 & 0.5227 & 0.5769 & 0.5812 \\
\hline LB94/AgK99 ${ }^{c}$ & 1.1461 & 1.1125 & 1.0582 & 1.0399 \\
\hline
\end{tabular}

${ }^{a}$ Refs: Sea58: Seaton (1958); SSS69: Saraph et al. (1969); ENS69: Eissner et al. (1969); ES74: Eissner \& Seaton (1974); Men83: Mendoza (1983); Ag83: Aggarwal (1983); BLS89: Burke et al. (1989); Ag93: Aggarwal (1993); LB94: Lennon \& Burke (1994); AgK99: Aggarwal \& Keenan (1999).

${ }^{b}$ Results from Aggarwal (1993).

${ }^{c}$ Collision strength ratio.

\section{D.2. Collisional excitation of [OIII]}

Effective collision strengths $\Omega$ obtained over the past 50 years are listed in Table D. 1 at four $T_{\mathrm{e}}$ for transitions ${ }^{3} \mathrm{P}-{ }^{1} \mathrm{D},{ }^{3} \mathrm{P}-{ }^{1} \mathrm{~S}$ and ${ }^{1} \mathrm{D}-{ }^{1} \mathrm{~S}$. Aggarwal \& Keenan (1999) did not feel it necessary to update earlier values by Aggarwal (1993; Ag93), almost contemporary with Lennon \& Burke (1994). The ratios of the recent values are given in Table D.1. The differences are over $4 \%$ for ${ }^{3} \mathrm{P}-{ }^{1} \mathrm{D}$ and $10 \%$ for ${ }^{1} \mathrm{D}-{ }^{1} \mathrm{~S}$ (6\% in I Zw 18 conditions), but the latter has no influence at low $N_{\mathrm{e}}$. NEBU includes a fit better than $0.5 \%$ to $\mathrm{Ag} 93$ data.

The [O III] transition probabilities used in NEBU are from Galavis et al. (1997, GMZ97). The accuracy of the Opacity Project (OP) data for these transitions is $8-10 \%$ (Wiese et al. 1996). Coherently, the much more elaborate results by GMZ97 differ from the OP results by $9.6 \%$ and $5.5 \%$ for $\mathrm{A}\left({ }^{1} \mathrm{D}-{ }^{1} \mathrm{~S}\right)$ and $\mathrm{A}\left({ }^{1} \mathrm{P}-{ }^{1} \mathrm{~S}\right)$ respectively. Would $\mathrm{A}\left({ }^{1} \mathrm{D}-{ }^{1} \mathrm{~S}\right)$ change by as much as $5 \%$, the branching ratio of [O III] $] 4363$ would change by $0.6 \%$. 


\section{Péquignot: Heating of IZw 18, Online Material p 6}

Thus, discrepancies not exceeding 5\% exist among different calculations (3\% for $\Omega$ ratios), suggesting that uncertainties on the computed $r([\mathrm{O} \mathrm{III}])$ are probably $<5 \%$. The $25-30 \%$ underestimation found by SS99 is not due to erroneous atomic data.

\section{D.3. Miscellaneous data}

The adopted table for radiative and dielectronic recombinations is limited to the 11 sequences that are H-like-Na-like (Badnell 2006). Dielectronic rates for [S II $]-[S$ IV] are given by Badnell (1991), but total recombination coefficients for (recombined ions) [Si II $],[\mathrm{S} \mathrm{II}],[\mathrm{S} \mathrm{III}],[\mathrm{Ar} \mathrm{V}],[\mathrm{Fe} \mathrm{II}]-[\mathrm{Fe} \mathrm{V}]$, are taken from Nahar and co-workers (Nahar 2000, and references cited). The recombination rate for $[\mathrm{S} \mathrm{II}]$ used in this and previous NEBU computations is 1.15 times the Nahar value. Empirical total rate coefficients based on planetary nebula models (Péquignot, unpublished), implemented in NEBU for a decade, are 5 and 8 times the radiative ones for $[\mathrm{Ar} \mathrm{II}]$ and $[\mathrm{Ar} \mathrm{III}]$ respectively. A larger factor is suspected for [Ar III] at high $T_{\mathrm{e}}$.

Collision strengths of special mention include those for [O II] (Pradhan et al. 2006; also Tayal 2006b), [O IV] (Tayal 2006), [S III] (Tayal \& Gupta 1999), [S IV] (Tayal 2000) and [Fe V] (Wöste et al. 2002). Collisions with $\mathrm{H}^{0}$ are considered in Sect. 6.5. Charge exchange rates with $\mathrm{H}^{0}$ for $\mathrm{O}^{2+}$ and $\mathrm{N}^{2+}$ are now from Barragán et al. (2006). 